\title{
IL-23 reshapes kidney resident cell metabolism and promotes local kidney inflammation
}

\author{
Hao Li, ${ }^{1}$ Maria G. Tsokos, ${ }^{1}$ Rhea Bhargava, ${ }^{1}$ Iannis E. Adamopoulos, ${ }^{1}$ Hanni Menn-Josephy, ${ }^{2}$ Isaac E. Stillman, ${ }^{3}$ Philip Rosenstiel, ${ }^{4}$ \\ Jarrat Jordan, ${ }^{5}$ and George C. Tsokos ${ }^{1}$ \\ 'Department of Medicine, Beth Israel Deaconess Medical Center, Harvard Medical School, Boston, Massachusetts, USA. 2Department of Medicine, Renal Section, Boston University School of Medicine, \\ Boston, Massachusetts, USA. ${ }^{3}$ Department of Pathology, Beth Israel Deaconess Medical Center, Harvard Medical School, Boston, Massachusetts, USA. Innstitute of Clinical Molecular Biology, Kiel University \\ and University Hospital Schleswig-Holstein, Campus Kiel, Kiel, Germany. ${ }^{5}$ Janssen Research \& Development, LLC, Spring House, Pennsylvania, USA.
}

\begin{abstract}
Interstitial kidney inflammation is present in various nephritides in which serum interleukin 23 (IL-23) is elevated. Here we showed that murine and human renal tubular epithelial cells (TECs) expressing the IL-23 receptor (IL-23R) responded to IL-23 by inducing intracellular calcium flux, enhancing glycolysis, and upregulating calcium/calmodulin kinase IV (CaMK4), which resulted in suppression of the expression of the arginine-degrading enzyme arginase 1 (ARG1), thus increasing in situ levels of free L-arginine. Limited availability of arginine suppressed the ability of infiltrating T cells to proliferate and produce inflammatory cytokines. TECs from humans and mice with nephritis expressed increased levels of IL-23R and CaMK4 but reduced levels of ARG1. TEC-specific deletion of II23r or Camk4 suppressed inflammation, whereas deletion of Arg1 exacerbated inflammation in different murine disease models. Finally, TEC-specific delivery of a CaMK4 inhibitor specifically curbed renal inflammation in lupus-prone mice without affecting systemic inflammation. Our data offer the first evidence to our knowledge of the immunosuppressive capacity of TECs through a mechanism that involves competitive uptake of arginine and signify the importance of modulation of an inflammatory cytokine in the function of nonlymphoid cells, which leads to the establishment of an inflammatory microenvironment. New approaches to treat kidney inflammation should consider restoring the immunosuppressive capacity of TECs.
\end{abstract}

\section{Introduction}

Interstitial inflammation is frequently present in patients with autoimmune kidney diseases (1). The presence of kidney-infiltrating immune cells, including monocytes $(2-4)$, B cells $(5,6)$, and T cells (7) has been linked to fibrosis and kidney failure (8), and thus is a well-recognized risk factor for morbidity and mortality (9). Intrarenal $\mathrm{T}$ cells recognize kidney-specific antigens and proliferate locally (10-12). Local microenvironmental factors, including hypoxia (13) and nutrient concentration $(14,15)$, which may be altered in inflammatory conditions, regulate the survival and pathogenesis of infiltrating T cells $(16,17)$. Cellular metabolism has emerged as an important determinant of $\mathrm{T}$ cell fate and function $(18,19)$. The competitive uptake of nutrients by tissue-resident cells may reshape the local immune response by altering the availability of nutrients (20), which implies that in kidneys, resident cells like tubular epithelial cells (TECs), mesangial cells, endothelial cells, and podocytes may function not only as victims but also active contributors to the development of renal inflammation (21).

Related Commentary: https://doi.org/10.1172/JCI150588

Conflict of interest: The authors have declared that no conflict of interest exists. Copyright: (5) 2021, American Society for Clinical Investigation.

Submitted: July 20, 2020; Accepted: May 5, 2021; Published: June 15, 2021.

Reference information: J Clin Invest. 2021;131(12):e142428.

https://doi.org/10.1172/JCl142428.
Interleukin 23 (IL-23) is a heterodimeric cytokine of the IL-12 superfamily (22) that exhibits a proinflammatory role through its ability to contribute to the generation of Th17 cells (23). Yet, it is unknown whether IL-23 may alter the function of nonlymphoid cells. The IL-23/IL-17 pathway has been reported to contribute to the pathogenesis of kidney disease characterized by chronic renal inflammation (24-27). Here we show that in the absence of additional inflammatory signals, IL-23 alone drives kidney inflammation characterized by massive T lymphocyte infiltration. Furthermore, genetic ablation of the IL-23 receptor (IL-23R) in renal TECs limited kidney inflammation in different models of murine renal disease.

Mechanistically, we show that IL-23 acts directly on TECs to initiate intracellular calcium flux and enhance the expression of calcium/calmodulin-dependent protein kinase IV (CaMK4) (28), which results in the suppression of the expression of the arginine-hydrolyzing enzyme arginase 1 (ARG1) (29). Arginine is a semi-essential amino acid that plays a central role in the regulation of the immune response and in its absence $\mathrm{T}$ cell proliferation is abolished (30, 31). Therefore, IL-23-mediated downregulation of ARG1 in TECs establishes an inflammatory milieu by providing arginine, which promotes local $\mathrm{T}$ cell activation and proliferation. Collectively, our results reveal that kidney TECs exert a local immunosuppressive role that is negated in the presence of IL-23.

\section{Results}

IL-23 is sufficient to instigate kidney inflammation. The elevation of serum IL-23 in patients with active systemic lupus erythema- 
A
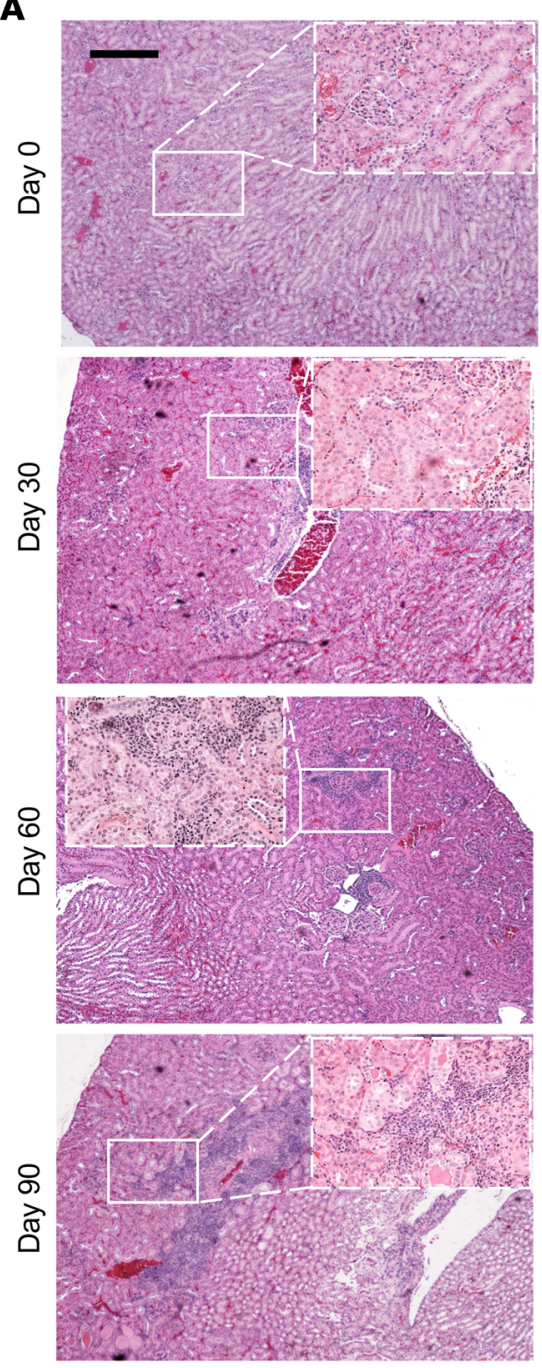

$H \& E \times 5$
C
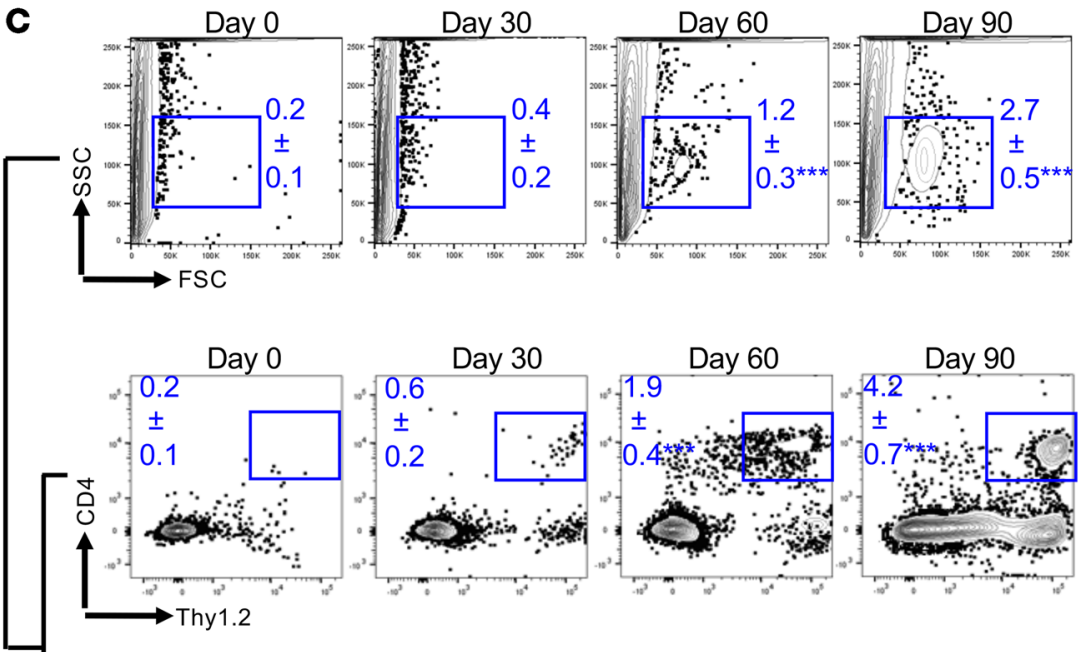
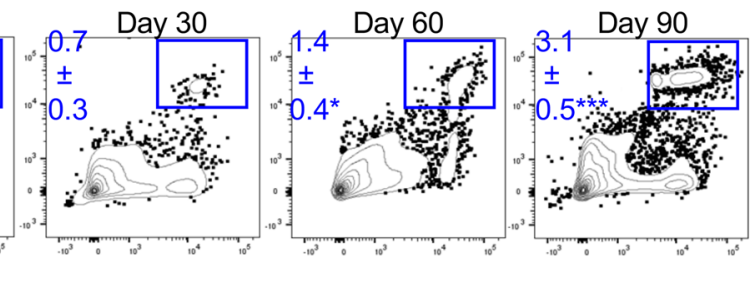

D
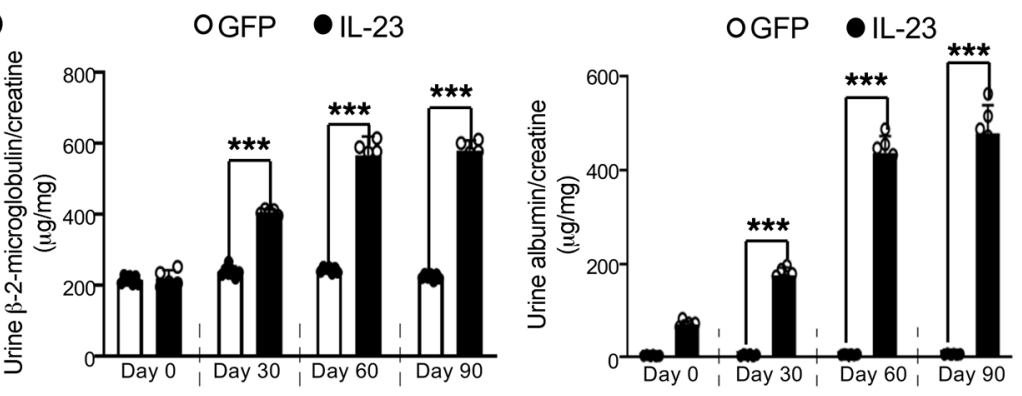

B

Day 0

Day 30

Day 60
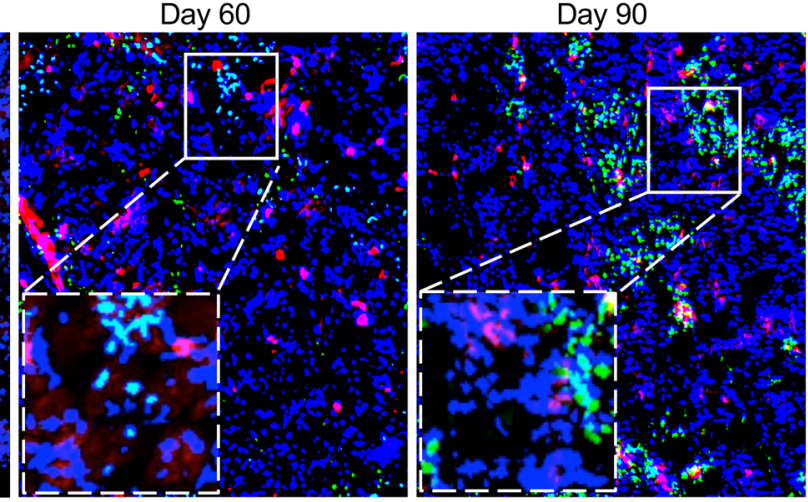

Figure 1. IL-23 is sufficient to instigate kidney inflammation. Eight-week-old B6 mice were injected with IL-23 minicircle (MC) for the indicated days (A-D), (A) Representative images of H\&E-stained kidney sections from the indicated mice; boxed areas were digitally magnified. Magnification, $\times 5$; scale bar: 200 $\mu \mathrm{m}$. (B) Cryosection of kidneys from the indicated mice stained for CD4+ $\mathrm{T}$ cells (green) and Gr-1+ macrophages/neutrophils (red); boxed areas were digitally magnified. Hoechst (blue) was used as nuclear counterstain. Magnification, $\times 10$; scale bar: $50 \mu \mathrm{m}$. (C) Flow cytometric quantification of infiltrating CD4 ${ }^{+} \mathrm{T}$ cells and $\mathrm{Gr}-1^{+}$macrophages/neutrophils in kidneys. (D) ELISA quantification of protein in urine from indicated mice. Left: Urine $\beta$-2-microglobulin from the indicated mice. Right: The mean ratio of urine albumin and creatinine from the indicated mice. All images are representative kidney regions. Data represent the mean \pm SEM; $n=5-7$ mice per group in each experiment for 2 independent experiments. ${ }^{*} P<0.05,{ }^{* * *} P<0.005$ vs. control by Student's $t$ test. 
tosus (SLE) plus the fact that this elevation preceded the appearance of proteinuria (Supplemental Figure 1; supplemental material available online with this article; https://doi.org/10.1172/ JCI142428DS1) affirmed the hypothesis that the IL-23/IL-17 axis contributes to the pathogenesis of various types of nephritis $(7,23$, 32); however, it remains unknown whether these cytokines could act on resident kidney cells directly. To investigate the role of IL-23 in renal inflammation, we overexpressed IL-23 in wild-type 8-week-old C57BL/6J (B6) mice by hydrodynamic administration of a minicircle (MC) cDNA construct encoding IL-23p19 and IL-12/23p40 (IL-23 MC), while GFP MC was used as control (Supplemental Figure 2) $(33,34)$. Hydrodynamically delivered IL-23 MC has been shown to induce psoriatic arthritis-like symptoms in male B10.RIII mice (33-35). As expected, skin inflammation and injury developed in these B6 mice receiving single-dose IL-23 MC (Supplemental Figure 3); however, arthritis was not observed. The discrepancy could be explained by different major histocompatibility complex (MHC) haplotypes. Interestingly, we further observed that in vivo expression of IL-23 in B6 mice was sufficient to phenocopy the tubulointerstitial inflammation component (Supplemental Figure 4 and Figure 1A) frequently documented in human nephritis, which predicts renal failure (8). Mice that received GFP MC did not develop tubulointerstitial inflammation (Supplemental Figure 4). Confocal image analysis revealed gradual accumulation of renal immune cell infiltration including $\mathrm{T}$ cells, activated monocytes, and neutrophils 30 days after IL-23 MC administration (Figure 1B). Analysis of renal immune cell infiltrates by flow cytometry confirmed the emergence of $\mathrm{CD} 4^{+} \mathrm{T}$ cells (Figure $1 \mathrm{C}$ and Supplemental Figures 4 and 5), at a time later than the accumulation of activated $\mathrm{CD}^{+} \mathrm{T}$ cells, especially Th17 cells, in the spleen as part of systemic inflammation (Supplemental Figure 6). T helper cells are important for activating and recruiting neutrophils and macrophages to the site of inflammation. As expected, accumulation of Gr- $1^{+}$activated macrophages and neutrophils in the tubulointerstitial space was also observed (Figure $1 \mathrm{C}$ and Supplemental Figures 4 and 5). Of note, proteinuria was observed in mice that received IL-23 MC but not GFP MC (Figure 1D). An increased ratio of urinary $\beta$-2-microglobulin and albumin to creatinine indicated renal tubular malfunction and podocyte failure (Figure 1D) (36).

Epithelial IL-23 signaling initiates calcium flux and promotes glycolysis. Having demonstrated that IL-23 alone can initiate and propagate renal inflammation, we asked whether there were kidney-resident cells responding to IL-23 prior to the development of renal inflammation. To address this question, GFP or IL-23 MC was administered to naive B6 mice for 24 hours only. When elevated circulating IL-23 but not renal immune cell infiltration was observed, kidneys were harvested to examine IL-23R protein expression on resident cells. Interestingly, elevated IL-23R expression was observed in kidneys from mice administered IL-23 MC but not those administered control GFP MC (Figure 2A). The antibody binding specificity was validated using polarized T cells (Supplemental Figure 7). Confocal images further confirmed the increase in IL-23R mainly on CD26+ TECs $(37,38)$ (Figure 2B). In order to comprehensively characterize IL-23-initiated signaling events in kidney-resident cells, primary TECs were sorted from B6 mice injected with either GFP or IL-23 MC (Supplemental Figure 8) and the mRNA expression levels of different genes were assessed using a customized PCR array, which revealed that TECs displayed a completely different gene expression pattern upon IL-23 stimulation in vivo. Notably, a number of IL-23-associated genes such as Stat3, Il23r, Jak2, and Socs3 were significantly enriched along with increased expression of Camk4 but not Camk2 (Figure 2C). To further validate the direct impact of IL-23 on TECs, proximal TECs were isolated from naive B6 mice and selectively enriched in vitro (39). The expression of IL-23-related genes was increased in TECs following stimulation with IL-23 in a time-dependent fashion (Figure 2D). Calcium-dependent mechanisms play pivotal roles in kidney function (40) and TECs are among the cells that are sensitive to cellular calcium changes (41). Interestingly, a modest but significant increase in calcium flux was observed in mouse primary renal proximal TECs stimulated with IL-23 (Figure 2E). Since calcium is integral in many important aspects of cell biology including cell metabolism, we quantified glycolysis and mitochondrial respiration through the measurement of the extracellular acidification rate (ECAR) and oxygen consumption rate (OCR), respectively $(42,43)$. Increased ECAR but not OCR was observed in IL-23-stimulated primary TECs (Figure 2F and Supplemental Figure 9). Interestingly, this enhancement was abrogated following the addition of EGTA, a chelating agent with a high affinity for calcium ions, which indicated that IL-23-boosted glycolysis was calcium dependent. Considering that $\mathrm{Ca}^{2+} /$ calmodulin-dependent protein kinase kinases (CaMKKs) are serine/threonine kinases activated by the binding of $\mathrm{Ca}^{2+} / \mathrm{CaM}$, which helps remove the autoinhibitory domain and enables substrate access (44), the IL-23-induced intracellular calcium flux might lead to enhanced CaMK4 expression and activation. To validate this supposition, mouse TECs cultured in the presence or absence of IL-23 were collected and analyzed by Western blotting. Indeed, increased IL-23R and CaMK4 but not CaMK2 was observed in TECs stimulated with IL-23 (Figure 2G), which was further confirmed by confocal image analysis (Figure $2 \mathrm{H}$ ). Furthermore, to extend our observations in vitro, the expression of CaMK2 and CaMK4 was assessed in the kidneys of mice administered GFP or IL-23 MC. As expected, immunohistochemical staining revealed a significant increase in CaMK4 but not CaMK2 in TECs from IL-23 MC-administered mice (Figure 2I). Collectively, our data indicate that TECs responded to IL-23 by upregulating CaMK4, which might control cellular processes related to immune functions.

Epithelial IL-23 signaling enables T cell proliferation in coculture. Although it is clear that IL-23 promotes the expansion of Th17 cells (45), it remains unknown whether IL-23R expression on TECs contributes to local inflammation. Amino acid metabolism is recognized as a critical factor in immune regulation and arginine is a semi-essential amino acid that serves as a substrate in numerous enzymatic pathways (46). Conversion of arginine by ARG1 can regulate $\mathrm{T}$ cell responses by controlling the bioavailability of this amino acid $(30,47)$. PCR arrays (Figure 2, C and D) revealed that stimulation of TECs with IL-23 reduced the expression of ARG1 both in vitro and in vivo, which led us to consider the possibility that expression of ARG1 in TECs constrains renal inflammation by reducing arginine levels in the surrounding environment. Accordingly, the IL-23-mediated downregulation of ARG1 should favor the activation and expansion of kidney-infiltrating immune cells, including $\mathrm{T}$ cells. To address this possibility, we cocultured CFSE-labeled purified splenic $\mathrm{CD}^{+} \mathrm{T}$ cells with TECs in arginine-deficient medium. 
A

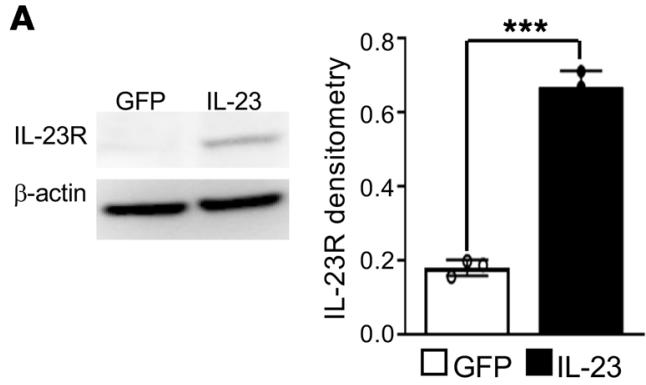

B
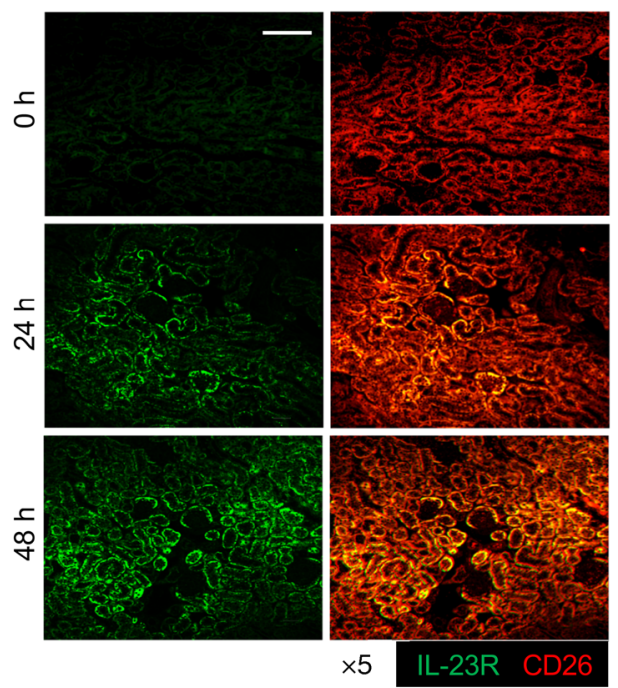

E

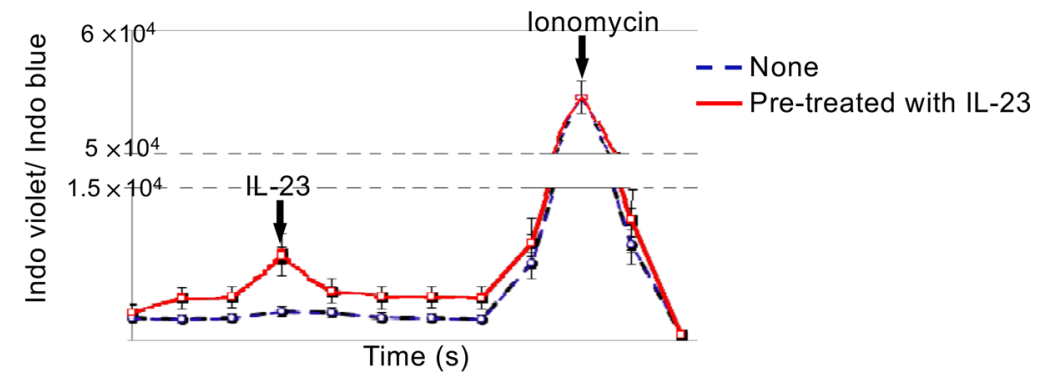

F

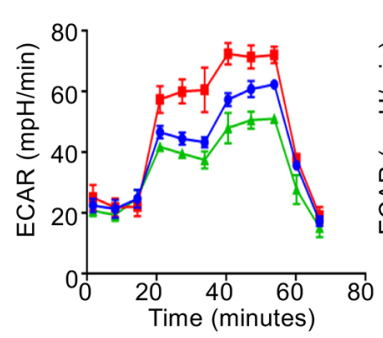

- Control $\square \mathrm{IL}-23 \Delta \mathrm{IL}-23+\mathrm{EGTA}$
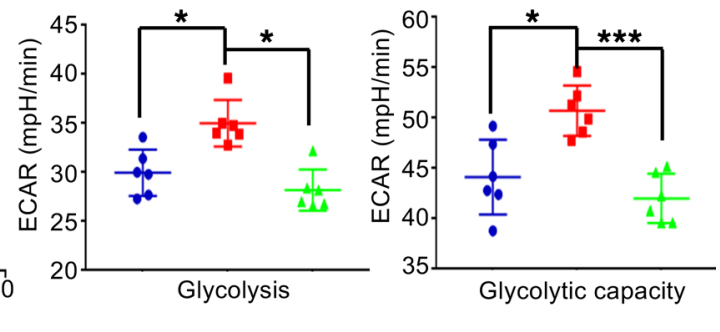

\section{G}

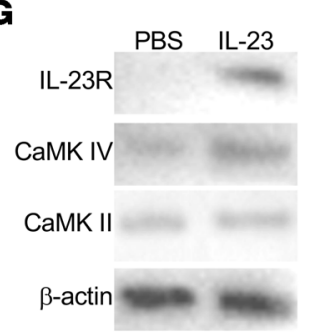

C

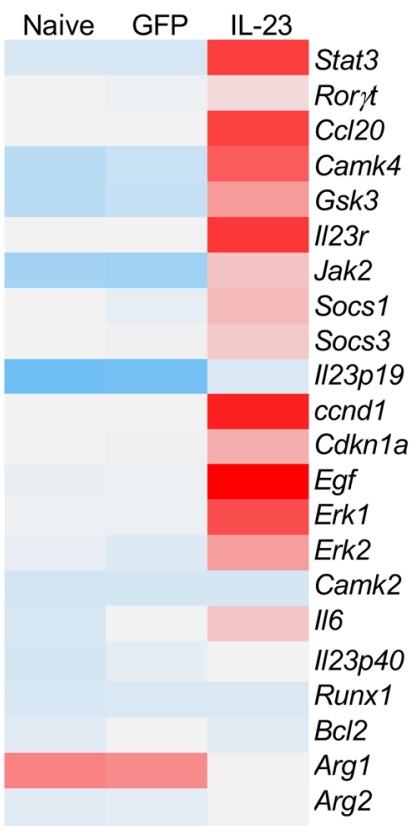

D

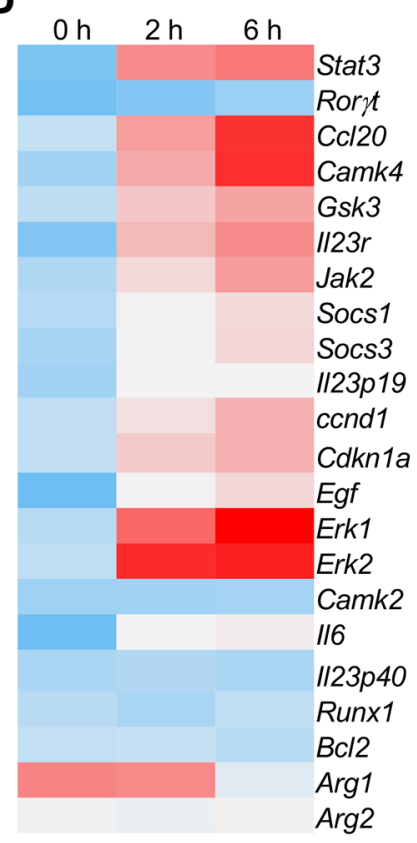

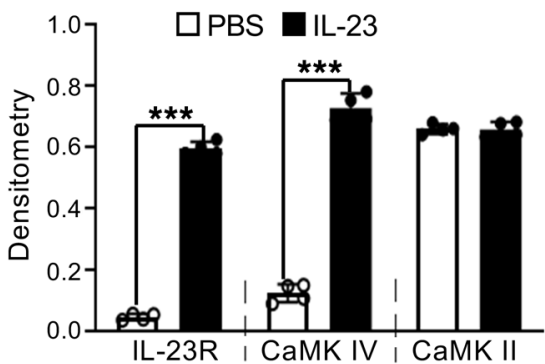

H

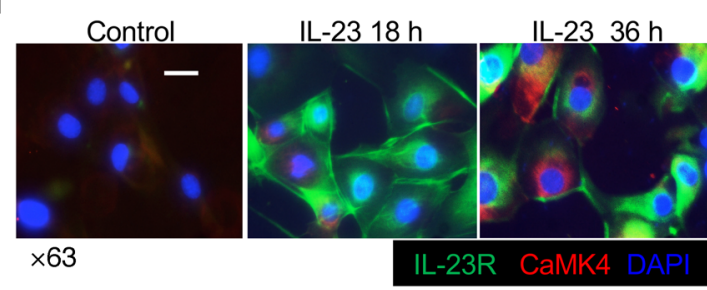

I

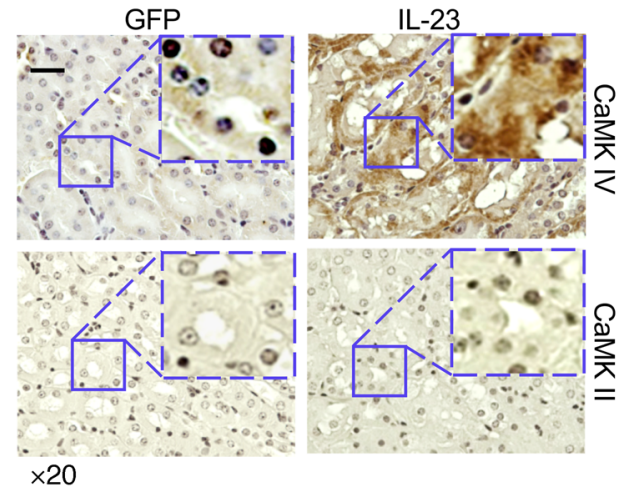


Figure 2. Epithelial IL-23 signaling initiates calcium flux and promotes glycolysis. (A) Western blots (left) and quantification (right) of IL-23R expression in total kidneys from B6 mice administered the indicated MC for 24 hours. (B) Cryosections of kidneys from mice subjected to IL-23 MC administration for the indicated time. Magnification, $\times 5$; scale bar: $120 \mu \mathrm{m}$. (C) Heatmap of PCR array showing differential expression of 22 genes in primary TECs sorted from naive mice or mice subjected to the indicated MC for 72 hours. Primary proximal TECs were isolated from B6 mice and selectively enriched in vitro (D-H). (D) Heatmap of PCR array showing differential expression of 22 genes in cultured TECs stimulated with IL-23 for the indicated hours. (E) Cumulative results of the mean fluorescence ratio of indo- 1 violet versus indo- 1 blue, which represent intracellular calcium flux. TECs were prestimulated with IL-23 for 6 hours and then restimulated with IL-23 (negative control: PBS; positive control: ionomycin). (F) Left: Representative mean ECAR of cultured TECs prestimulated with IL-23 for 6 hours with or without addition of EGTA and unstimulated cells as control. Dot plots represent cumulative data of calculated glycolysis (middle) and glycolytic capacity (right). (C) Western blots (left) and quantification (right) of the expression of the indicated proteins in TECs stimulated with IL-23 or PBS for 48 hours. (H) Immunofluorescence image analysis of IL-23R (green) and CaMK4 (red) expression in TECs stimulated with IL-23 for the indicated hours (DAPI as nuclear counterstain, blue). Magnification, $\times 63$; scale bar: $10 \mu \mathrm{m}$. (I) Representative images of immunohistochemical (IHC) staining of CaMK4 (upper) or CaMK2 (lower) in kidneys from mice subjected to the indicated MC for 2 months; boxed areas were digitally magnified. Magnification, $\times 20$; scale bar: $25 \mu \mathrm{m}$. ${ }^{*} P<0.05,{ }^{* *} P<0.005$ vs. control by Student's $t$ test. $n$ $=4-6$ per group in each experiment for 2 independent experiments.

As expected, in the absence of arginine, $\mathrm{T}$ cells lost the ability to proliferate (31) and addition of arginine restored $\mathrm{T}$ cell proliferation in the absence but not in the presence of TECs (Figure 3A). Interestingly, in the coculture system, IL-23 efficiently removed the TEC-dependent immunosuppression and supported $\mathrm{T}$ cell activation and proliferation (Figure $3 \mathrm{~A}$ and Supplemental Figure 10). Activated $\mathrm{T}$ cells can take up citrulline to generate arginine, which helps reconstitute $\mathrm{T}$ cell proliferation in the absence of arginine (48). We examined whether adding citrulline to the coculture system could rescue $\mathrm{T}$ cell proliferation. As expected, the addition of citrulline partially released the exogenous constraint on $\mathrm{T}$ cell activation and proliferation by TECs (Figure 3A and Supplemental Figure 10). Thus, the competition for arginine was important for TEC-mediated immunosuppression and IL-23 could reverse this suppression by downregulating the expression of ARG1.

To validate the reduction in ARG1 at the protein level, proximal TECs were isolated from Arg1-eYFP mice and cultured in vitro. After stimulation with IL-23, but not IL-17, both confocal microscopy and flow cytometry revealed the downregulation of ARG1 (Figure 3B). Interestingly, the CaMK4 inhibitor KN93 (49) reduced or even reversed the IL-23-mediated downregulation (Figure 3C), which strongly suggested that CaMK4 functioned upstream of ARG1 in the IL-23-stimulated TECs. To confirm this signaling cascade in TECs, we generated Cdh16-Cre $\times I l 23 r^{f l / f l}\left(I l 23 r^{\Delta \mathrm{TEC}}\right)$, Cdh16Cre $\times \operatorname{Camk}^{f l / f l}\left(\operatorname{Camk}^{\Delta \mathrm{TEC}}\right)$, and Cdh16-Cre $\times \operatorname{Arg} 1^{f / / f l}\left(\operatorname{Arg} 1^{\Delta \mathrm{TEC}}\right) \mathrm{B} 6$ mice (Cre recombinase expression is detected in renal tubules of adult mice) (50). As expected, in Arg1 $1^{\triangle \mathrm{TEC}} \mathrm{B} 6$ mice, ARG1 expression was markedly reduced in renal interstitium but not glomeruli (Supplemental Figure 11). Next, proximal TECs were isolated, enriched, and cultured in vitro for protein expression analysis (39). Consistent with the above results, IL-23 stimulation upregulated IL-23R and CaMK4, but not CaMK2, and downregulated ARG1 (Figure 3, D and E). Of note, deficiency of Camk4 efficiently pre- vented the IL-23-mediated downregulation of ARG1, which confirmed that CaMK4 was required for the subsequent reduction in ARG1 (Figure 3, D and E). To confirm our hypothesis that IL-23 signaling in TECs is involved in augmenting the immune response, we cocultured CFSE-labeled purified splenic $\mathrm{CD} 4^{+} \mathrm{T}$ cells with different TECs in arginine-complete medium. TECs suppressed T cell proliferation in the coculture system. ARG1 expression was essential for this immunosuppression and the presence of IL-23 negated this suppression, which was dependent on CaMK4 (Figure 3F). To confirm our findings in vivo, we injected IL-17 or IL-23 MC into Arg1-eYFP mice. IL-23 but not IL-17 MC administration reduced ARG1 expression in TECs in renal interstitium in vivo (Figure 3G and Supplemental Figure 12). Interestingly, coinjection of KN93 (i.p.) efficiently prevented IL-23-mediated ARG1 reduction in vivo (Figure 3H). Noteworthily, the expression of ARG1 in glomeruli was unaffected. Collectively, these data demonstrate that IL-23 modulated the immune-regulatory capacity of TECs by enhancing CaMK4 expression, which subsequently suppressed ARG1 expression and made arginine available locally.

Genetic deficiency of Il23r or Camk4 ameliorates, but deficiency of Arg1 exaggerates, renal inflammation in mice. To confirm our findings in vivo, we administered IL-23 MC to Il23r $r^{\triangle \mathrm{TEC}}$, Cam$k 4^{\triangle \mathrm{TEC}}$, or Arg1 ${ }^{\triangle \mathrm{TEC}} \mathrm{B} 6$ mice, while Cdh16-Cre mice were used as controls (50). The serum levels of IL-23 were comparable in all groups (Supplemental Figure 13). Failed induction of either IL-23R or CaMK4 in TECs was observed in $I l 23 r^{\triangle \mathrm{TEC}}$ or Camk $4^{\Delta \mathrm{TEC}}$ mice (Supplemental Figure 14). As expected, histological and flow cytometric assessment confirmed the reduction of both percentages and absolute numbers of intrarenal infiltrating $\mathrm{CD} 4^{+} \mathrm{T}$ cells and neutrophils in $I l 23 r^{\triangle \mathrm{TEC}}$ and Camk $4^{\triangle \mathrm{TEC}}$ mice (Figure 4, A-C, and Supplemental Figure 15). By contrast, the intrarenal infiltrating $\mathrm{CD}^{+} \mathrm{T}$ cells and neutrophils were increased in Arg1 $1^{\triangle \mathrm{TEC}}$ mice compared with Cdh16-Cre control mice (Figure 4, A-C, and Supplemental Figure 15). Consistently, deficiency of either Il23r or Camk4 in TECs ameliorated, but deficiency of Arg1 exaggerated, IL-23 MC-induced proteinuria (Figure 4D).

Of note, the splenic Th17 cells were almost equivalent in all 4 groups (Supplemental Figure 16) despite the fact that IL-23R and CaMK4 are essential for Th17 cells, which further validated the targeting specificity of Cdh16-Cre. To test our hypothesis in experimental models that mimic human kidney diseases, toxic sheep anti-mouse glomeruli serum was administered into Cdh16-Cre control, Il $23 r^{\Delta \mathrm{TEC}}$, and Camk $4^{\triangle \mathrm{TEC}}$ mice $(100 \mu \mathrm{L} /$ mouse) to induce inflammation in kidneys, which resembled human anti-glomerular basement membrane (anti-GBM) nephritis (51). Twenty-one days after serum injection, Cdh16-Cre mice exhibited pronounced glomerulonephritis with crescent formation, as well as tubulointerstitial inflammation (Figure 4E) along with severe proteinuria (Supplemental Figure 17). Flow cytometric analysis revealed the presence of infiltrating $\mathrm{CD} 4^{+} \mathrm{T}$ cells, activated macrophages, and neutrophils in inflamed kidneys (Figure 4E). Deficiency of $I l 23 r$ or Camk4 in TECs ameliorated proteinuria and kidney pathology, as manifested by the reduced mesangial cell proliferation and crescent formation, and the presence of inflammatory cells (Figure 4, E-G, and Supplemental Figure 18). To fully evaluate the ARG1-mediated immunosuppressive capacity of TECs, we reduced the volume of toxic sheep serum to the extent that induced only mild 
A No Arginine Arginine Arginine + Citrulline
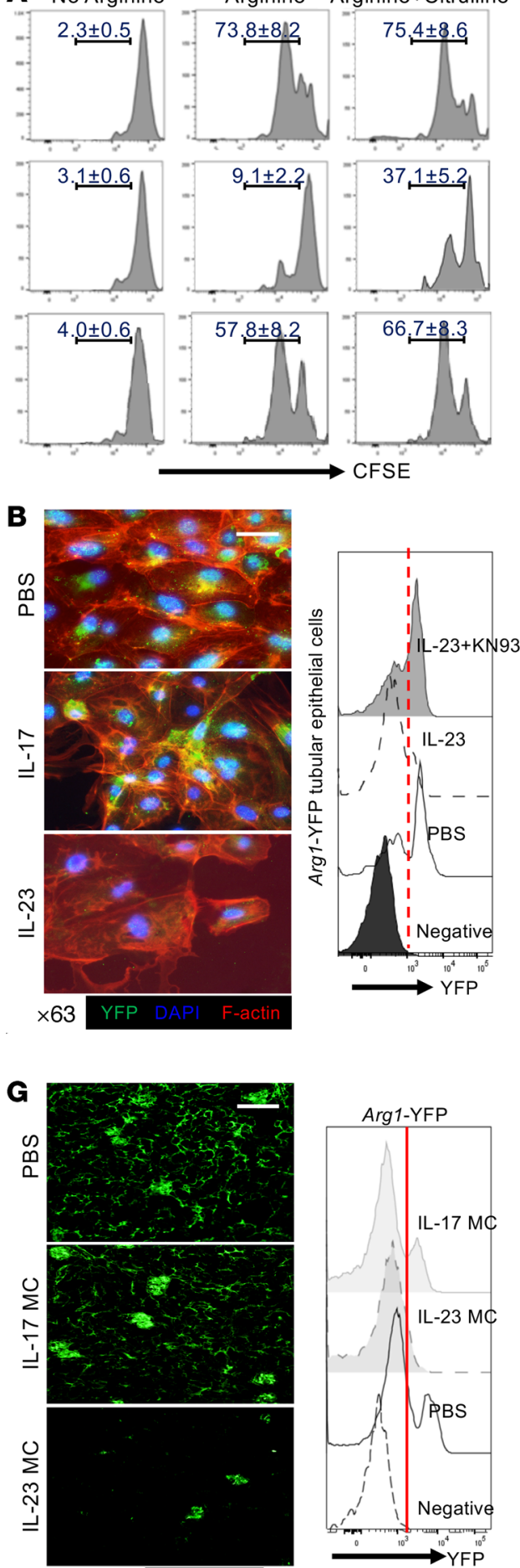

×5 $\operatorname{Arg1-YFP}$

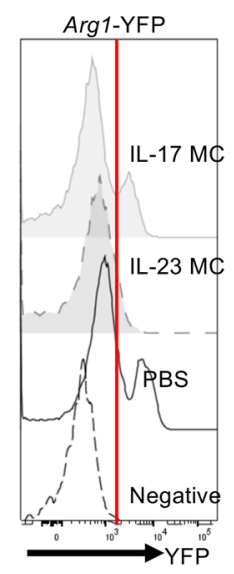

D

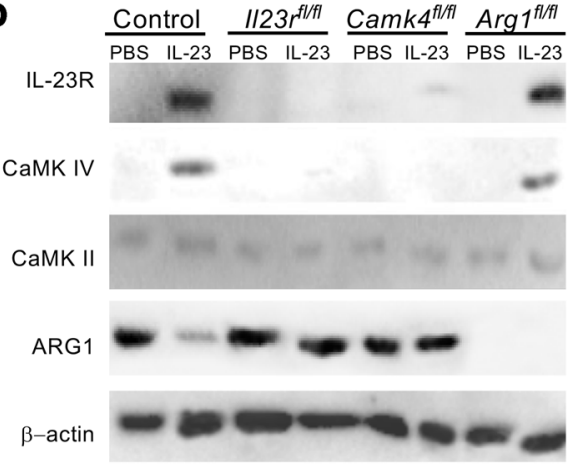

C
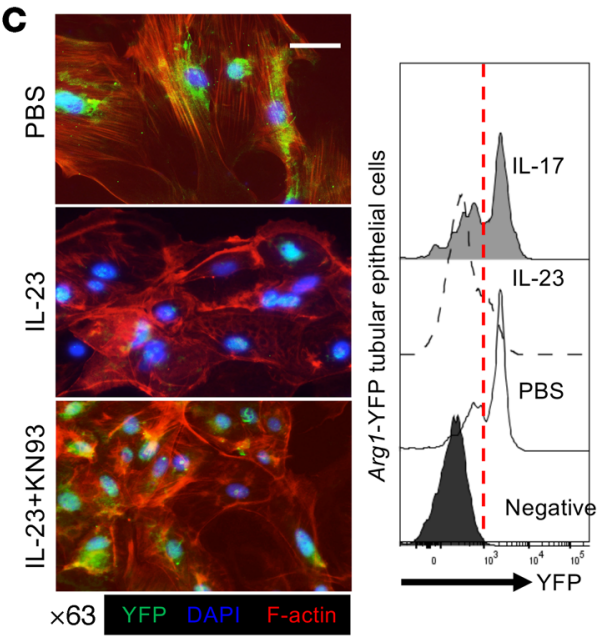

H
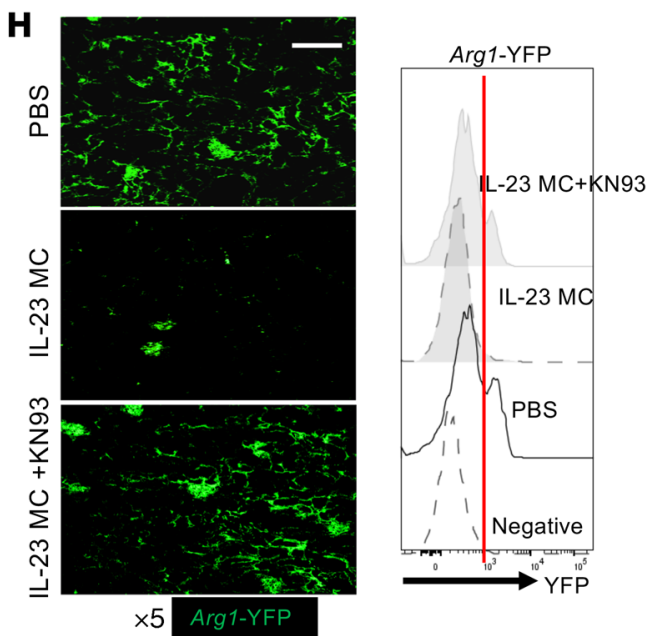
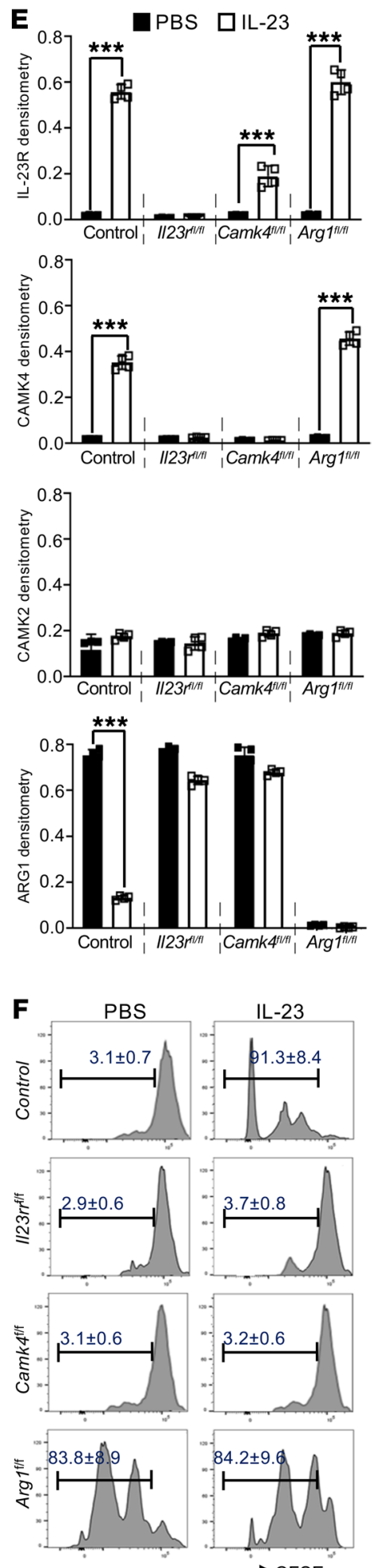
Figure 3. Tubular epithelial IL-23 signaling enables $\mathrm{T}$ cell proliferation in coculture. (A) Flow cytometric analysis of in vitro proliferative response (CFSE ${ }^{10}$ ) of purified splenic CD4 ${ }^{+} \mathrm{T}$ cells cocultured with primary TECs or IL-23-prestimulated TECs for 72 hours in arginine-deficient media with or without addition of arginine or citrulline. T cells were stimulated with anti-CD3 and anti-CD28. Primary proximal TECs were isolated from Arg1-eYFP B6 mice and selectively enriched in vitro. ARG1 expression was tracked by fluorescence intensity of YFP. (B and C) Left: Immunofluorescence image analysis for YFP expression (ARG1) with phalloidin-counterstained F-actin (red) and DAPI (blue) in cultured TECs with the indicated cytokine stimulation (B) or stimulated in the presence of IL-23 for 72 hours with or without CaMK4 inhibitor (KN93) and PBS as control (C). Magnification, $x 63$; scale bar: $10 \mu \mathrm{m}$. Right: Flow cytometric quantification of ARG1 expression. Primary proximal TECs were isolated from mice with the indicated gene deficiency and selectively enriched in vitro. All mice were Cdh16-cre+. Cells were stimulated with IL-23 or PBS for 48 hours for Western blots (D) and quantification (E) of the expression of the indicated proteins. (F) Flow cytometric analysis of in vitro proliferative response $\left(\mathrm{CFSE}^{10}\right.$ ) to anti-CD3 plus anti-CD28 of purified splenic CD4 ${ }^{+} \mathrm{T}$ cells cocultured 72 hours with the indicated proximal TECs with or without IL-23 stimulation. Arg1-eYFP B6 mice were injected with the indicated MC for 48 hours and KN93 was i.p. injected 2 hours after MC administration. ( $\mathbf{G}$ and $\mathbf{H}$ ) Left: Representative confocal microscopic images of renal YFP (ARG1) for mice subjected to the indicated MC administration (C) or mice administered IL-23 MC with or without addition of KN93 (H). Magnification, $\times 5$; scale bar: $150 \mu \mathrm{m}$. Right: Flow cytometric quantification of ARG1 expression; cells from naive B6 mice were used as negative control. Data represent the mean \pm SEM; $n=4-6$ per group in each experiment for 2 independent experiments. ${ }^{* * *} P<0.005$ by Student's $t$ test.

nephritis in control Cdh16-Cre mice and applied this dose to $\operatorname{Arg} 1^{\triangle \mathrm{TEC}}$ mice. Not surprisingly, the deficiency of Arg1 in TECs promoted renal inflammation and injury characterized by increased immune cell infiltration and exaggerated immunopathology (Figure 4, H-J, and Supplemental Figures 17 and 18). Together, these results support the immunosuppressive capacity of TECs and the IL-23/CaMK4 axis-instigated immunopathogenesis in chronic kidney inflammation.

TEC-targeted delivery of a CaMK4 inhibitor efficiently suppresses renal inflammation without affecting systemic inflammation in lupus-prone MRL.lpr mice. Nephritis is a common manifestation in people with SLE (52). MRL.lpr mice are frequently used to study SLE because they develop many lupus manifestations, including nephritis (53). Serological examination affirmed that the age-associated elevation of IL-23 in the sera preceded the appearance of proteinuria (Supplemental Figure 19), which was consistent with our above observation in SLE patients. Although numerous studies have delineated the pathogenic role of the IL-23/Th17 axis in SLE $(27,54)$, it is not known whether IL-23 affects nonhematopoietic cells. Having demonstrated the possible involvement of IL-23 signaling in TEC-propagating kidney inflammation, we asked whether this axis contributes to lupus nephritis. Similar to our observation above, serological examination affirmed that the age-associated increase in IL-23 in MRL.lpr mice preceded the appearance of proteinuria. Immunofluorescence and immunohistochemical staining revealed that age-associated increases in IL-23R and CaMK4 expression aligned with a gradual reduction in ARG1 levels in TECs of MRL.lpr mice (Figure 5, A-C). Considering the fact that CaMK4 is widely expressed in many types of cells $(55,56)$, we aimed to determine whether inhibition of CaMK4 in a TEC-specific manner could prevent kidney inflammation and injury in lupus-prone mice. To accomplish this, we explored the possibility of delivering KN93 in a cell-targeted manner by loading the drug into nanolipogels tagged with a TEC-specific antibody against cadherin-16 $(55,57)$. To test the delivery specificity of the antibody-coated nanolipogels in vivo, we injected rhodamine-labeled nanolipogels, tagged with anti-cadherin-16, into 10-week-old MRL.lpr mice. Confocal images revealed that there was a time-dependent renal TEC-specific enrichment of administered nanolipogels, which started at 15 minutes and peaked at 30 minutes after nanolipogel administration (Figure 5D). As expected, rhodamine was not detected in podocytes or other tissues. In order to assess the independent contribution of CaMK4 in TECs to the pathogenesis of lupus nephritis, KN93-loaded nanolipogels coated with anti-cadherin-16 was administered to female MRL.lpr mice weekly for 6 weeks starting at 10 weeks of age. The treatment did not mitigate systemic inflammation, including numbers of splenic Th1 and Th17 cells (Supplemental Figure 20) along with serum autoantibody titers (Supplemental Figure 21). However, the targeted delivery of KN93 produced a substantial therapeutic effect on proteinuria and kidney pathology, as manifested by reduced mesangial cell proliferation and crescent formation compared with the control group of animals administered anti-cadherin-16 antibody-tagged empty nanolipogels (Figure 5, E-H). Moreover, the flow cytometric analysis confirmed suppressed renal inflammation manifested by diminished numbers of infiltrating CD $4^{+} \mathrm{T}$ cells (Figure 5I).

To comprehensively assess the immunopathogenesis of the IL-23 signaling cascade in TECs for lupus nephritis, a series of bone marrow $(\mathrm{BM})$ reconstitution experiments were carried out in which BM from 2-month-old B6.lpr mice was transferred to lethally irradiated Cdh16-Cre control, Il23r $r^{\Delta \mathrm{TEC}}$, Camk $4^{\Delta \mathrm{TEC}}$, or Arg1 ${ }^{\triangle \mathrm{TEC}} \mathrm{B} 6$ recipients. In this experiment, epithelial cells, which are resistant to radiation-induced death, were of recipient origin, whereas hematopoietic cells were of donor origin (58). Similarly, deficiency of either Il23r or Camk4 in TECs impeded, whereas deficiency of Arg1 in TECs magnified, the immune cell infiltration into the kidneys without affecting splenic pathogenic T cells (Figure 5, J-L, and Supplemental Figures 22-24). Taken together, our results suggest the immunopathogenesis of the IL-23/CaMK4 axis in lupus nephritis.

Upregulation of $I L-23 R$ and CaMK4 and reduction of ARG1 in renal biopsies from patients with glomerulonephritis. Next, we asked whether we could replicate our findings using human samples. Human proximal TECs were cultured in vitro for ECAR and OCR assessment. Similar to our findings in mice, increased ECAR, but not OCR, was observed in IL-23-stimulated primary TECs and this augmentation was calcium dependent (Figure 6A and Supplemental Figure 25). Furthermore, expression of IL-23-linked genes in cultured human TECs was examined by quantitative PCR and the pattern of gene expression changes was almost identical to the one we observed in mouse TECs (Figure 6B). Western blot assay further supported that IL-23 stimulation upregulated IL-23R and CaMK4 and downregulated ARG1 (Figure 6, C and D). Consistently, silencing CAMK4 but not CAMK2 efficiently prevented the downregulation of ARG1 in TECs following stimulation with IL-23 (Figure 6, C and D). To validate the immunosuppressive capacity of TECs in humans, 

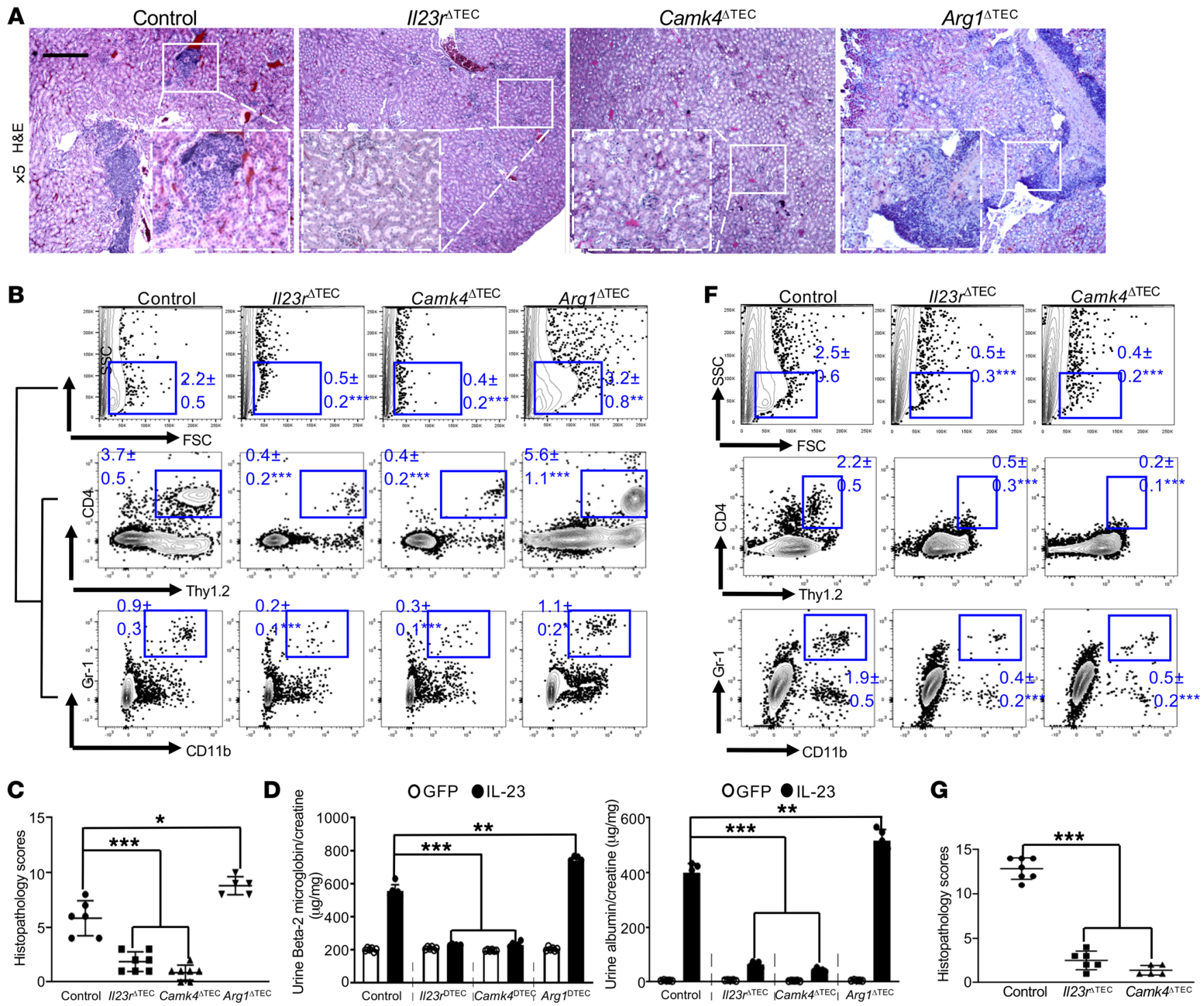

G
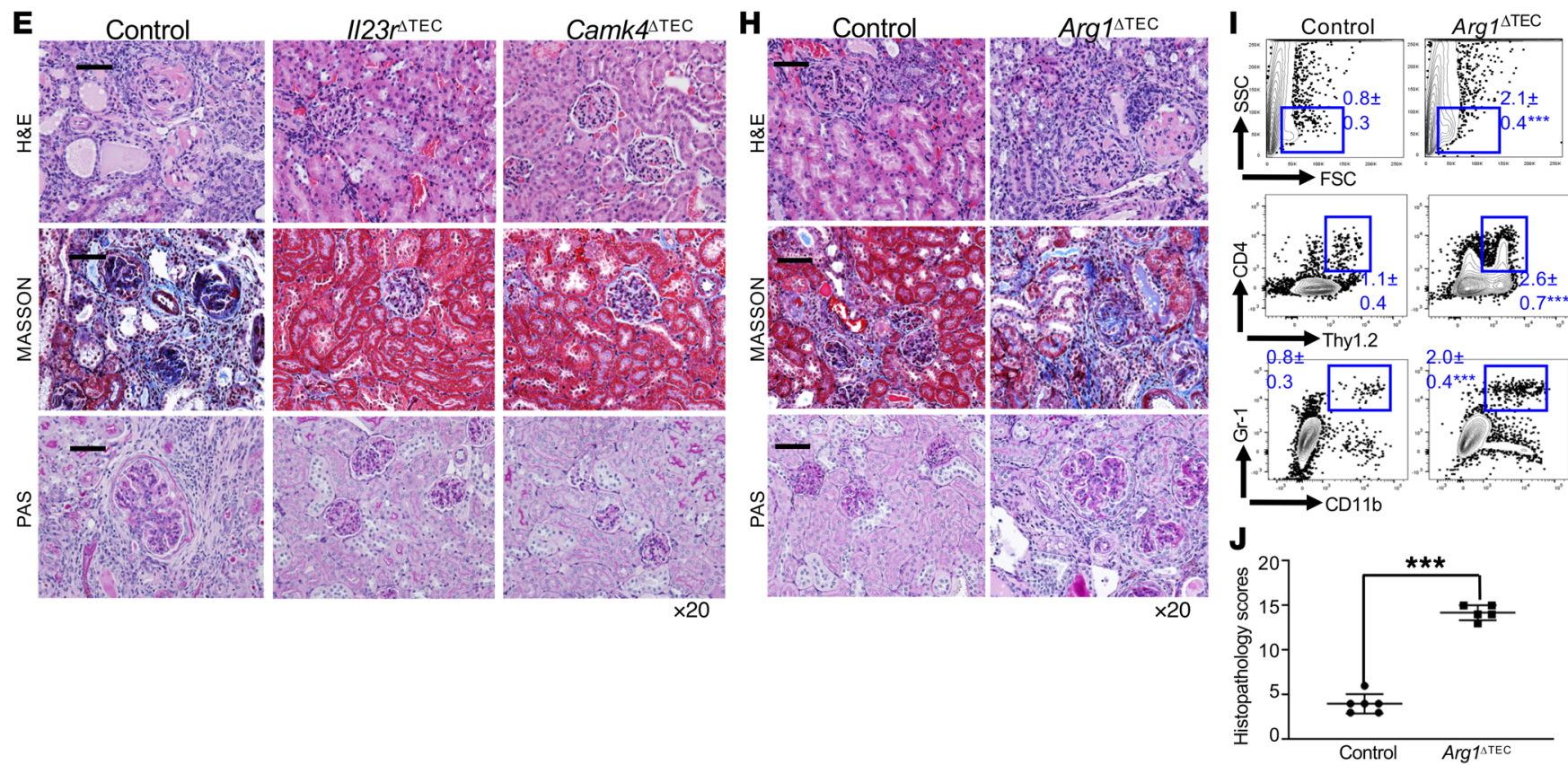
Figure 4. Genetic deficiency of II23r or Camk4 ameliorates, but deficiency of Arg1 exaggerates, renal inflammation in mice. All mice were Cdh16-cre+: Cdh16-Cre (control), Cdh16-Cre $\times I / 23^{f l / f f}\left(I / 23 r^{\Delta T E C}\right)$, Cdh16-Cre $\times$ Camk $4^{f l / f l}$

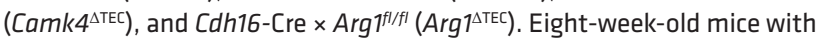
the indicated genotype were injected with IL-23 MC for 90 days (A-C). (A) Representative images of H\&E-stained kidney sections from the indicated mice; boxed areas were digitally magnified. Magnification, $x 5$; scale bar: $200 \mu \mathrm{m}$. (B) Flow cytometric quantification of infiltrating CD4 ${ }^{+} \mathrm{T}$ cells and activated macrophages/neutrophils in kidneys. (C) Histopathologic scoring of kidneys from the indicated mice. (D) ELISA quantification of protein in urine from the indicated mice. Left: Urine $\beta$-2-microglobulin from the indicated mice. Right: The mean ratio of urine albumin and creatinine from the indicated mice. ${ }^{* *} P<0.01,{ }^{* *} P<0.005$ versus control by 2 -way ANOVA. Sheep anti-mouse glomerular basement membrane (anti-GBM) serum (100 $\mu \mathrm{L}$ in $\mathbf{E}-\mathbf{C}$ or $50 \mu \mathrm{L}$ in $\mathbf{H - J}$ ) was administered to 8-week-old mice with the indicated genotype for 21 days for the induction of immune-mediated glomerulonephritis. (E) Representative images of H\&E, PAS, and Masson's trichrome staining of kidneys from the indicated 3 groups of mice subjected to sheep anti-mouse GBM serum. Magnification, $\times 20$; scale bar: $40 \mu \mathrm{m}$. (F) Flow cytometric quantification of infiltrating $\mathrm{CD}^{+} \mathrm{T}$ cells and activated macrophages/neutrophils in kidneys. (C) Histopathologic scoring of kidneys from the indicated mice. (H) Representative images of H\&E, PAS, and Masson's trichrome staining of kidneys from the indicated 2 groups of mice subjected to sheep anti-mouse GBM serum. Magnification, $\times 20$; scale bar: $40 \mu \mathrm{m}$. (I) Flow cytometric quantification of infiltrating CD4 ${ }^{+} \mathrm{T}$ cells and activated macrophages/neutrophils in kidneys. (J) Histopathologic scoring of kidneys from the indicated mice. Data represent the mean \pm SEM; $n=5-7$ mice per group in each experiment for 2 independent experiments. ${ }^{*} P<0.05,{ }^{*} P<0.01,{ }^{* *} P<0.005$ versus control by Student's $t$ test.

we cocultured CFSE-labeled purified peripheral blood CD4 ${ }^{+} \mathrm{T}$ cells with TECs in an arginine-deficient medium. As expected, in the absence of arginine, $\mathrm{T}$ cells significantly lost the ability to proliferate (Figure 6E). Addition of arginine restored $\mathrm{T}$ cell proliferation in the absence but not in the presence of TECs (Figure $6 \mathrm{E})$. However, IL-23 could help recover the capacity of T cells to proliferate in the absence of TECs (Figure 6E). Of note, TECs with CAMK4 deficiency were still capable of suppressing $\mathrm{T}$ cell proliferation after IL-23 stimulation (Figure 6E). Thus, activation of the IL-23/CaMK4 axis in human TECs perturbed their immunosuppressive functions.

To establish the clinical relevance of our findings, we examined the expression of IL-23R, CaMK4, and ARG1 in renal biopsy material from patients with various kidney inflammatory diseases (Supplemental Tables 1-3). The expression of IL-23R and CaMK4 was detected in samples from patients with active lupus nephritis $(n=13)$, anti-neutrophil cytoplasmic autoantibody-associated glomerulonephritis $(n=4)$, and renal transplant rejection $(n=7)$, but not normal kidney tissues $(n=5)$ (Figure $6 \mathrm{~F}$ ). After focusing on the renal interstitium, we noted increased expression of IL-23R and CaMK4 together with reduced expression of ARG1 on TECs but not on other cells (Figure 6, G and H, and Supplemental Figure 26). Next, we directed our attention to different types of lupus nephritis. Without a doubt, there was enhanced expression of both IL-23R and CaMK4 aligned with reduced ARG1 in TECs from patients with active lupus nephritis, either class III $(n=5)$ or class IV $(n=8)$. Impressively, kidney biopsy material from 4 lupus nephritis patients who achieved remission after treatment did not display increased expression of CaMK4 or decreased levels of ARG1 (Figure 6, I-K).

\section{Discussion}

Here we demonstrate that the proinflammatory cytokine IL-23 initiates local kidney inflammation by acting directly on resident cells. The process involves the upregulation of CaMK4, a serine/ threonine kinase, and the downregulation of an arginine-hydrolyzing enzyme, ARG1. Limited expression of ARG1 in TECs results in increased local availability of arginine, a key amino acid enabling $\mathrm{T}$ cell activation and proliferation (31). Inflammation has both systemic and local manifestations (4). Study of immune cells at the systemic level may not reflect with fidelity the events at the site of injury. Although the contribution of local factors in the development of inflammation has been considered, there is a general paucity of information on the direct effects of factors of systemic inflammation like circulating cytokines on tissue-resident cells such as kidney TECs. Our results provide the first evidence to our knowledge that TECs respond to IL-23, a systemically produced cytokine, through metabolic changes, which leads to the reshaping of the local microenvironment and ultimately enables the development of a local inflammatory response.

Even though the presence of a systemic inflammatory response manifested by the elevation of soluble inflammatory mediators including cytokines, chemokines, or antibodies in the circulation precedes and contributes to organ-specific inflammation (59), it remains unknown how these factors communicate with local resident cells to initiate or maintain tissue inflammation. Here, we show that IL-23, a causative factor for kidney inflammation, primes TECs to serve arginine to infiltrating $\mathrm{T}$ cells and support their proliferation (31). Furthermore, genetic ablation of either Il23r or Camk4 eliminated the development of kidney interstitial inflammation and targeted delivery of a CaMK4 inhibitor to TECs suppressed nephritis without affecting the systemic autoimmune response. Traditional therapies for chronic inflammatory diseases have relied on immunosuppressive medications that dampen the immune response systemically and thus increase susceptibility to life-threatening opportunistic infections $(52,59)$. Although IL-23 is a component of the systemic inflammatory response, it is needed for the instigation and the sustenance of organ inflammation (22), involving distinct factors and processes that can be manipulated independently.

Local environmental factors including nutrient availability $(16,20)$ and hypoxia (13) determine the establishment and sustenance of organ inflammation. Kidney tissue hypoxia, present inevitably in lupus nephritis, enables the sustenance of proinflammatory T cells (13). Nutrients, including amino acids, glucose, and fatty acids, determine immune cell metabolism $(14,20)$, which in turn dictates effector immune cell function (18). We have demonstrated the ability of ARG1 to enable kidney TECs to maintain an immunosuppressive environment by limiting the local availability of arginine, a process that is reversed by IL-23. Tubulointerstitial inflammation predicts disease outcome in various forms of nephritis and it has been proposed that TECs might actively contribute to the production of various inflammatory factors that propagate the injury locally or even in distant organs $(8,60)$. Activated T cells and inflammatory cytokines originating from the interstitium may migrate or diffuse freely and it is tempting to speculate that tubulointerstitial inflammation may initiate glomerular pathology, although it is wise to wait for additional experimental evidence for the crosstalk between interstitium and glomeruli. 

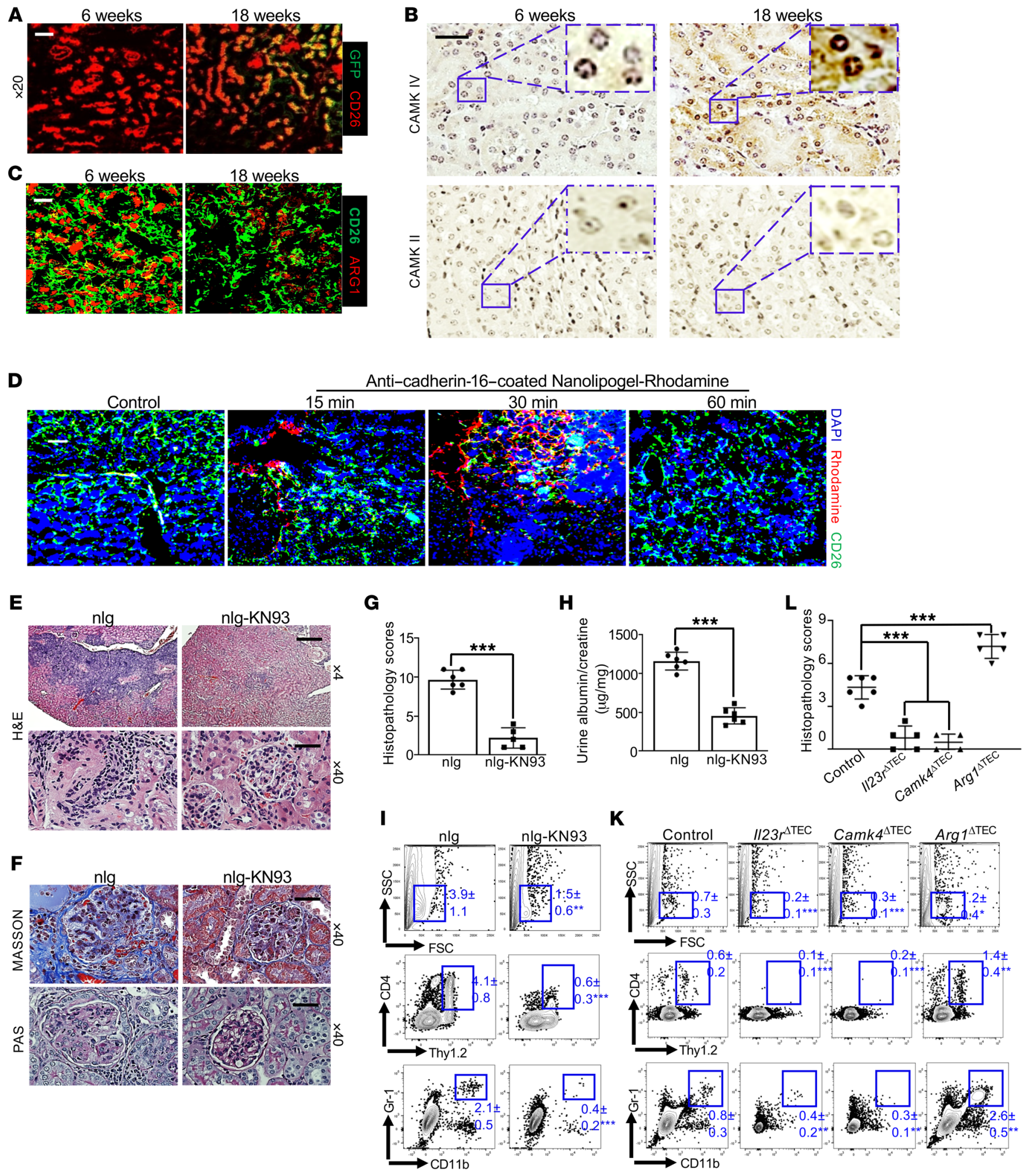

\section{$\mathbf{K}$}
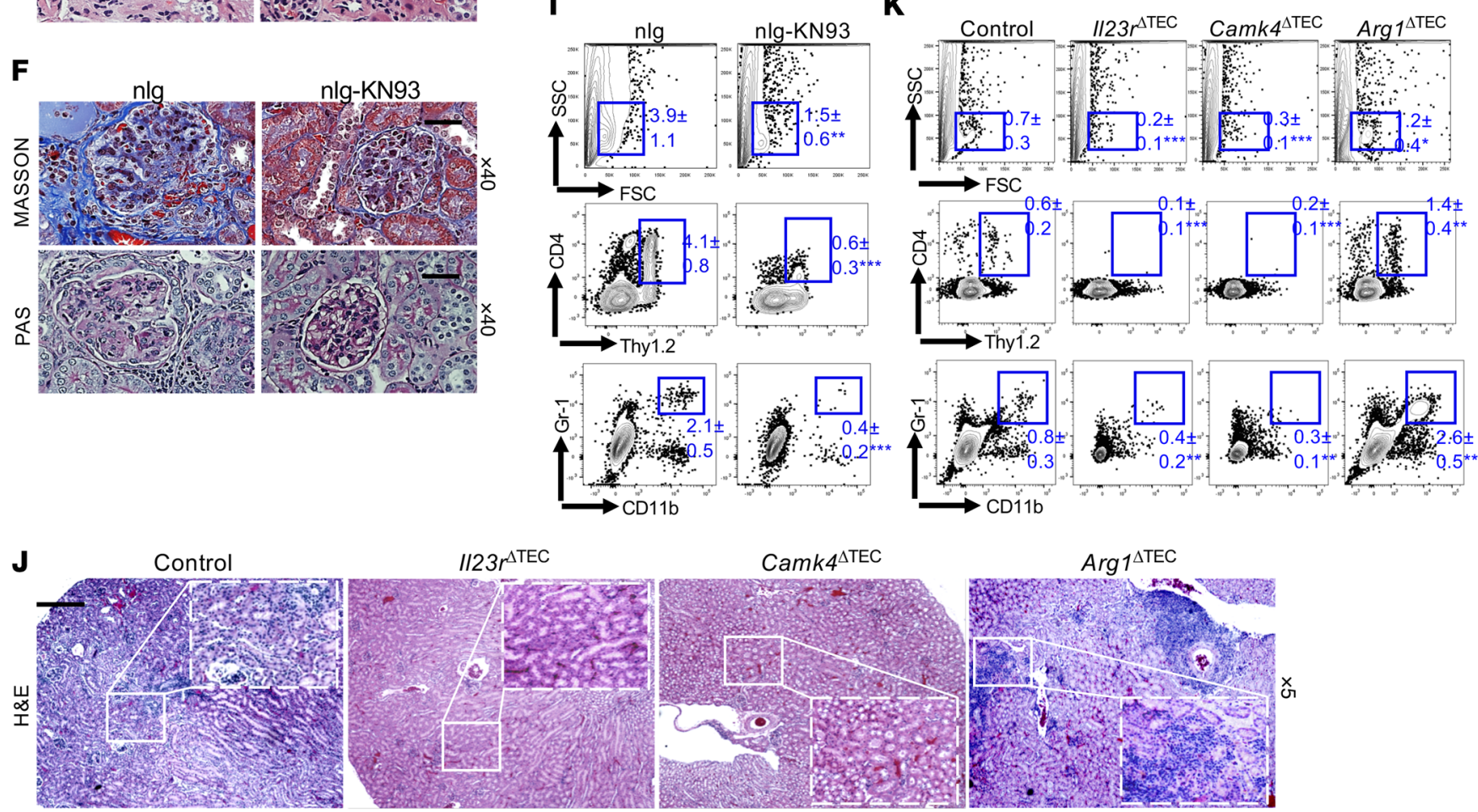
Figure 5. TEC-targeted delivery of the CaMK4 inhibitor KN93 efficiently suppresses renal inflammation in lupus-prone MRL.Ipr mice. (A) Cryosection of kidneys from female I/23r-GFP MRL.Ipr mice at the indicated ages stained for CD26 (red). Magnification, $\times 20$; scale bar: $20 \mu \mathrm{m}$. (B) Immunohistochemical staining of CaMK4 (left) and CaMK2 (right) in the kidneys of female MRL.Ipr mice at the indicated ages; boxed areas were digitally magnified. Magnification, $\times 40$; scale bar: $25 \mu \mathrm{m}$. (C) Cryosection of kidneys from in MRL.Ipr mice at the indicated ages stained for ARG1 (red) and CD26 (green). Magnification, $\times 20$; scale bar: $20 \mu \mathrm{m}$. (D) Ten-week-old MRL.Ipr mice were administered anti-cadherin-16-coated nanolipogel-rhodamine and euthanized after the indicated time for analysis. Confocal microscopic images show the targeted delivery by rhodamine ${ }^{+}$cells in representative kidney areas. Magnification, ×10; scale bar: $50 \mu \mathrm{m}$. (E-I) Ten-week-old MRL.Ipr mice were administered anti-cadherin-16-coated empty nanolipogels ( $\mathrm{nlg}$ ) or nanolipogel-KN93 every 10 days for a total of4 times. Representative images of kidney sections stained with $H \& E(E)$ and Masson's trichrome and PAS (F) with the indicated magnification. Scale bar: $200 \mu \mathrm{m}$ ( $\times 4$ magnification) and $25 \mu \mathrm{m}$ ( $\times 40$ magnification). (C) Histopathologic scoring of kidneys. (H) The mean ratio of urine albumin and creatinine. (I) Flow cytometric quantification of infiltrating $C D 4^{+} T$ cells and activated macrophages/neutrophils in kidneys. (J-L) Bone marrow (BM) reconstitution was carried out by transferring BM from 2-month-old B6.Ipr mice to lethally irradiated indicated recipients for 10 months. All recipients were Cdh16-cre': Cdh16-Cre (control), Cdh16-Cre $\times 1 / 23 r^{\text {fllfl }}\left(\right.$ (II23r $\left.{ }^{\Delta T E C}\right)$, Cdh16-Cre $\times$

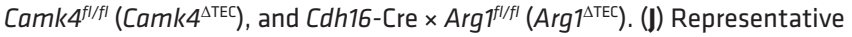
$\mathrm{H} \& \mathrm{E}$ images of kidneys from the indicated mice; boxed areas were digitally magnified. Magnification, $\times 5$; scale bar: $200 \mu \mathrm{m}$. (K) Flow cytometric quantification of $\mathrm{CD} 4^{+} \mathrm{T}$ cells and activated macrophages/neutrophils in kidneys. (L) Histopathologic scoring of kidneys. Data represent the mean \pm SEM; $n=4-6$ mice per group in each experiment for 2 independent experiments. ${ }^{*} P<0.05,{ }^{* *} P<0.01,{ }^{* * *} P<0.005$ versus control by Student's $t$ test ( $\mathbf{G}$ and $\mathbf{H}$ ) or 2-way ANOVA (K and $\mathbf{L}$ ).

Studies using kidney biopsies from patients with nephritis are limited by the number and the size of tissue samples. Other potential limitations are that activation of CaMK4 and the reduction of ARG1 may result from other factors associated with nephritis, such as elevated IL- 6 and TNF. Additionally, the downregulation of ARG1 in TECs could be due to treatment with cytotoxic drugs. Therefore, additional studies of human kidney biopsies are required to confirm the present results. IL-23 has been implicated in the immunopathogenesis of a series of inflammatory diseases (22), and monoclonal antibodies targeting different subunits of IL-23 have been demonstrated to have significant therapeutic value in the treatment of psoriasis, Crohn's disease, ulcerative colitis, and SLE with a better safety profile than conventional immunosuppressive therapies (61-63). Additionally, targeting IL-23p19 completely avoids effects on the IL-12/Th1 axis, essential for the clearance of intracellular pathogens, to minimize the risk of infections (22). However, mechanistic studies to assess whether IL-23 alters the function of nonimmune cells to educate immune cells are lacking. Our findings demonstrate that IL-23 can alter the metabolism of TECs, which results in a milieu conducive to $\mathrm{T}$ cell proliferation. These results should encourage additional studies on the influence of cytokines on the tissue microenvironment, which may reveal new tissue-specific targets of intervention for a variety of autoimmune diseases.

\section{Methods}

Mice. MRL.lpr, B6.lpr, wild-type B6, Thy1.1, Rag1-/, Cdh16 ${ }^{\mathrm{cre}+/}, \mathrm{Arg}^{\mathrm{eYFP}+}$, and $A r g 1^{f / f l}$ transgenic mice were obtained from The Jackson Laboratory. Camk $4^{f / f l}$ mice were maintained in our in-house mouse colony. Il-23r-
GFP-transgenic mice were provided by Mohamed Oukka (University of Washington and Children's Research Institute, Seattle, Washington, USA) (64). Il23r $r^{f / f l}$ mice were generated by as described previously (65). $I l 23 r^{f / f l}, \operatorname{Arg} 1^{f / f l}$, and Camk $4^{f / f l}$ mice were crossed with $C d h 16^{\mathrm{cre}+/+}$ mice to generate $C d h 16^{\mathrm{cre+}} \mathrm{Il} 23 \mathrm{r}^{\mathrm{fl} / \mathrm{fl}}, \mathrm{Cdh} 16^{\mathrm{cre}+} \mathrm{Arg} \mathrm{1}^{\mathrm{fl} / \mathrm{l}}$, and $\mathrm{Cdh} 16^{\mathrm{cre+}} \mathrm{Camk} 4^{\mathrm{ll} /}$ ${ }^{f l}$ mice. All mice were maintained under specific pathogen-free conditions at the animal facilities of the Beth Israel Deaconess Medical Center, Harvard Medical School.

BM transplantation. BM cells were harvested from B6.lpr mice and transferred into the indicated recipient mice $\left(2 \times 10^{7}\right.$ per mouse $)$ via i.v. injection immediately after irradiation.

Preparation of MC DNA constructs and MC administration in vivo. The MC DNA constructs were generated as described previously (33, 66). Briefly, a single isolated colony from a fresh plate was grown for the first 8 hours in Luria-Bertani broth with ampicillin, inoculated into Terrific broth for further expansion, and grown for an additional 17 hours. Subsequently, the medium was replaced with fresh Luria-Bertani broth containing 1\% L-arabinose. After adding one-half volume of fresh lowsalt Luria-Bertani broth ( $\mathrm{pH} 8.0$ ) containing 1\% L-arabinose, the incubation temperature was increased to $37^{\circ} \mathrm{C}$ and the incubation continued for 2 additional hours. Episomal DNA circles were prepared from bacteria using QIAGEN Endofree Megaprep plasmid purification kits. Hydrodynamic delivery of $8 \mu \mathrm{g}$ MC DNA per mouse was performed via tail vein injection in a total volume of approximately $10 \%$ of the mouse body weight within a period of 5-7 seconds in all experiments. Eightweek-old mice were used and sustained high levels of targeted gene expression for over 2 months in vivo were achieved by one injection.

Experimental anti-GBM disease in mice. Female 10- to 12-week-old B6 mice were primed with $0.5 \mathrm{mg}$ of sheep $\operatorname{IgG}$ in CFA 3 days prior to anti-GBM injection (67). Sheep anti-GBM antiserum was prepared as described previously (67), adsorbed with mouse erythrocytes (1:1), and titrated for optimized dose without immediate toxicity before use in i.v. injections. Mice were harvested on day 21 for assessment of histology and cellular infiltration by flow cytometry.

Histology of frozen sections. Tissues were embedded in OCT compound (Tissue-Tek) and frozen on dry ice. Frozen sections $(5 \mu \mathrm{m}$ thickness) were fixed to slides in ice-cold acetone for 15 minutes and air dried for 30 seconds. The sections were blocked with $10 \%$ horse serum for 30 minutes at room temperature and then stained for 30 minutes at room temperature in a humidified chamber with fluorescently labeled antibody cocktails. The following antibodies or conjugates with Fluor dyes were applied according to the manufacturers' instructions: mouse anti-human/mouse CaMK4 antibody (clone 26, BD Biosciences), mouse anti-human/mouse CaMK2 antibody (clone 45, BD Biosciences), anti-mouse CD4 (clone GK1.5, BioLegend), anti-mouse Gr-1 (clone RB6-8C5, BioLegend), anti-IL-23R (catalog number ab53656, Abcam) (58), and mouse anti-human/mouse ARG1 (clone O94E5, BD BioLegend).

Histology of paraffin-embedded tissue sections. Renal cross sections were fixed in $4 \%$ paraformaldehyde, embedded in paraffin, and deparaffinized in xylene, and then $4-\mu \mathrm{m}$ sections were stained with hematoxylin and eosin (H\&E), periodic acid-Schiff (PAS), and Masson's trichrome. For immunohistochemical staining, after heat-induced epitope retrieval and suppression of endogenous peroxidase activity by incubating the slides in $3 \% \mathrm{H}_{2} \mathrm{O}_{2}$ for 5 minutes, the sections were incubated overnight with purified mouse anti-human/mouse CaMK4 antibody (clone 26, BD Biosciences) and mouse anti-human/mouse 

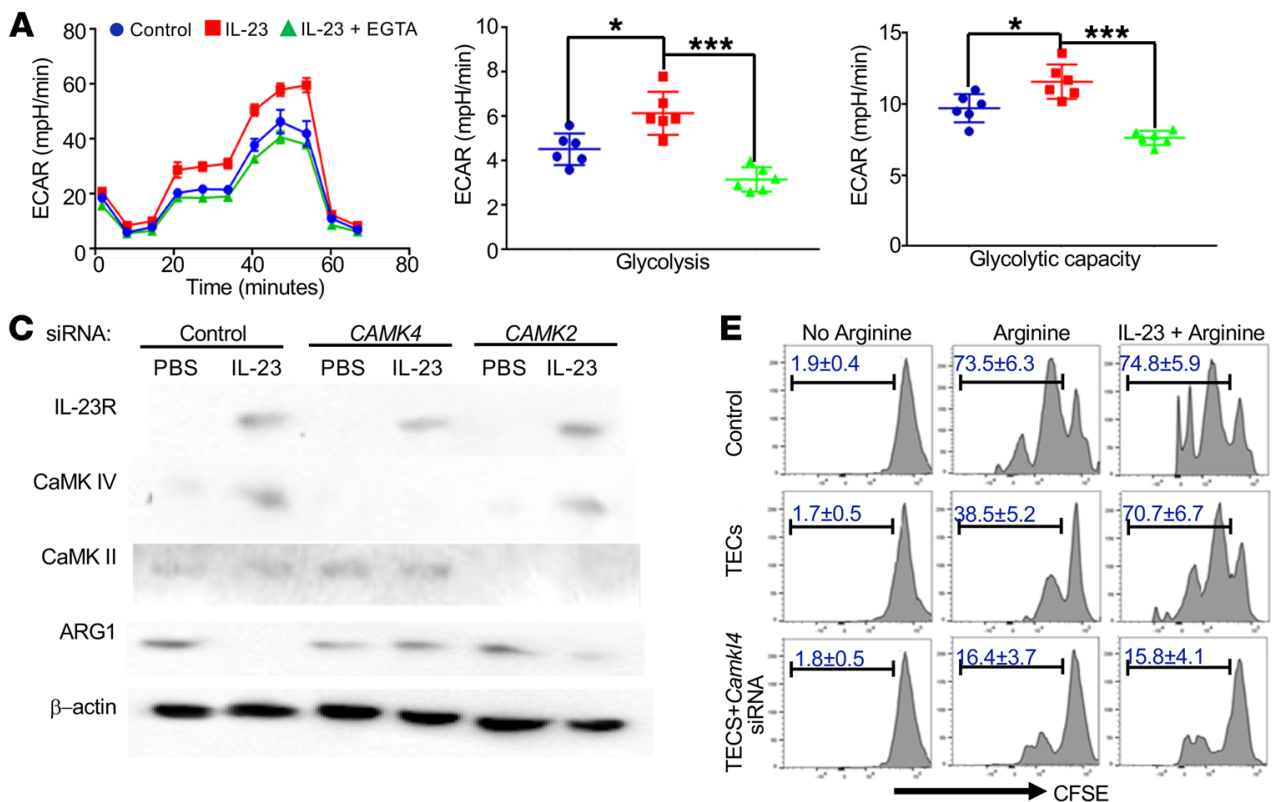
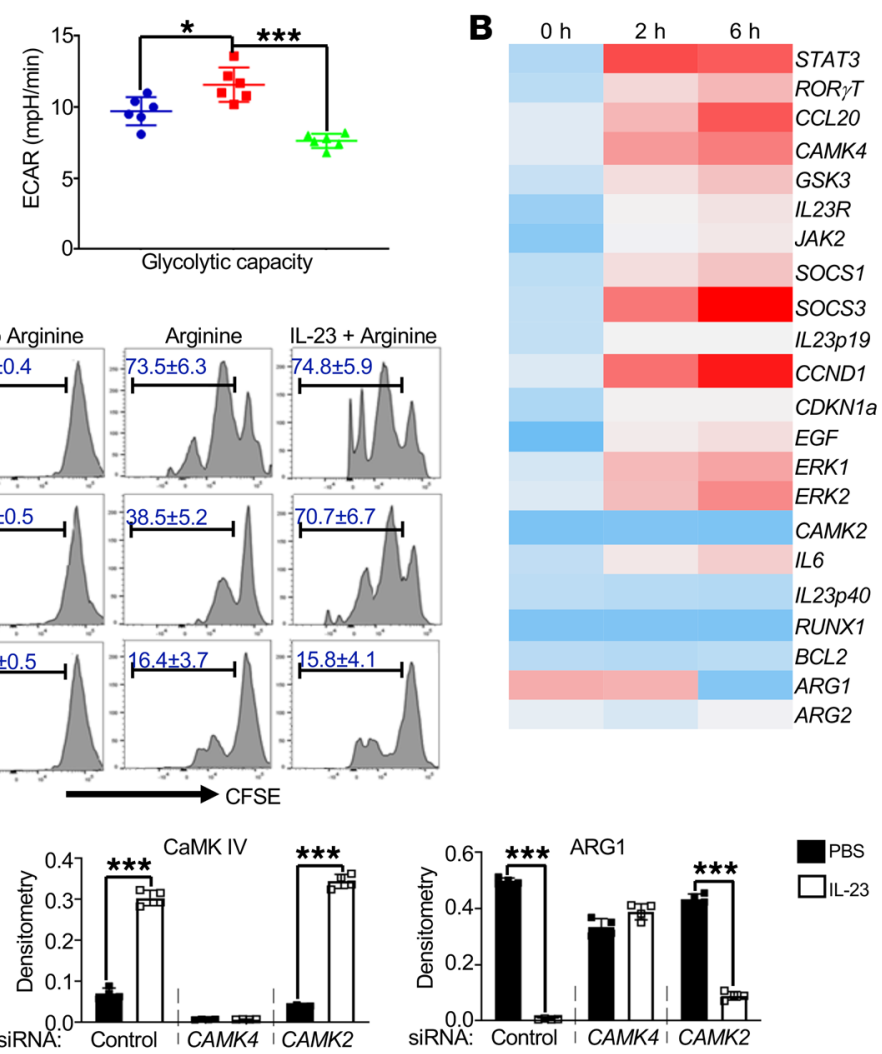
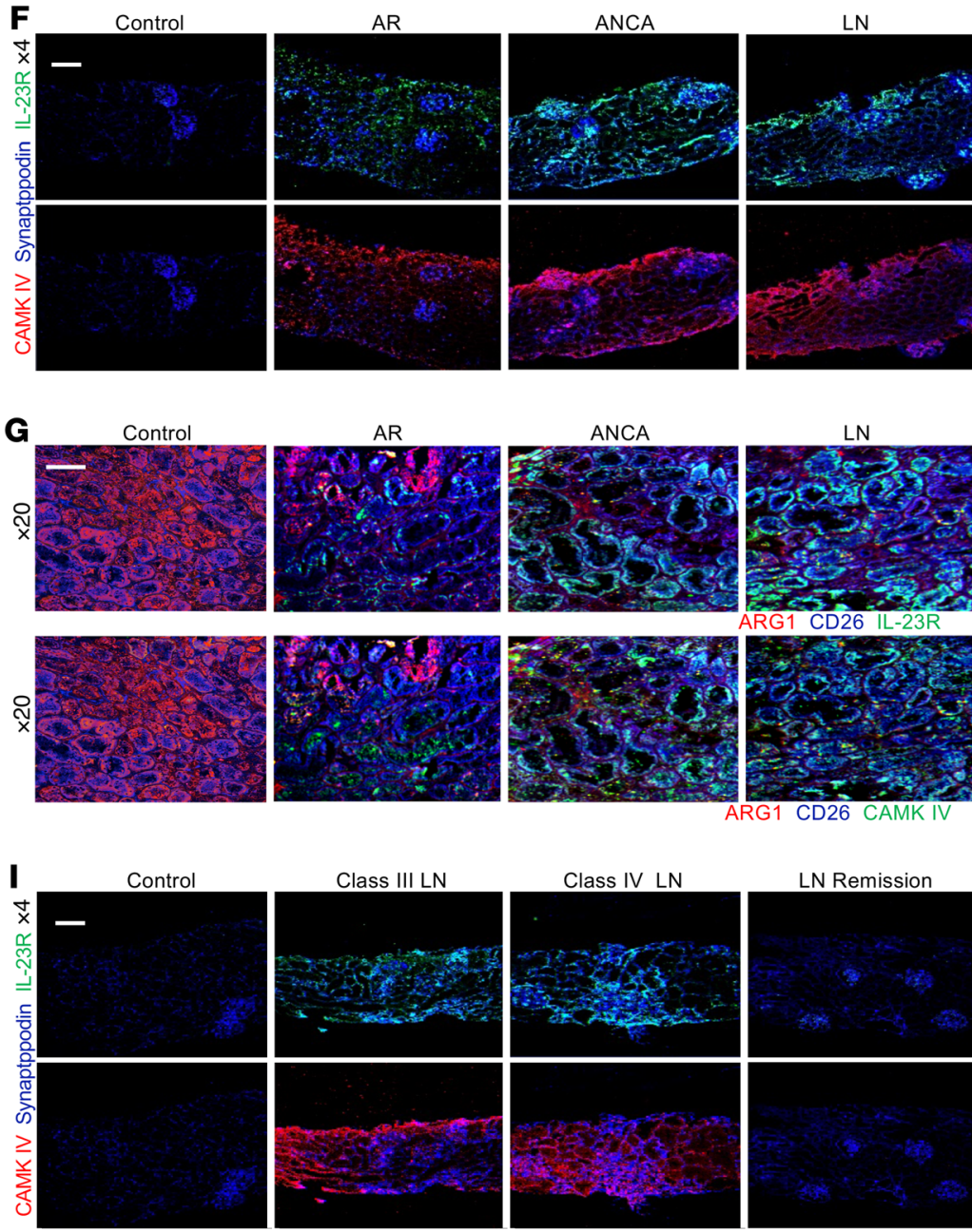

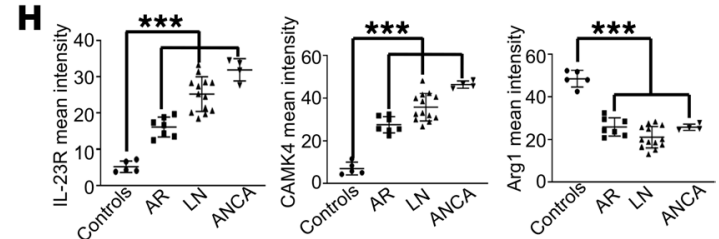

J
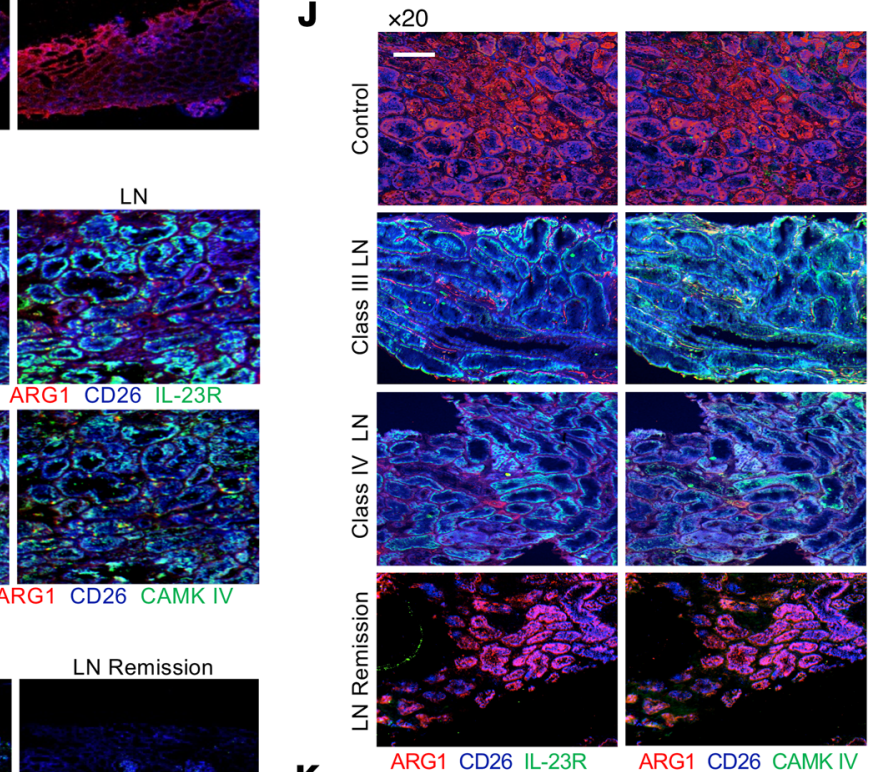

$\mathbf{K}$

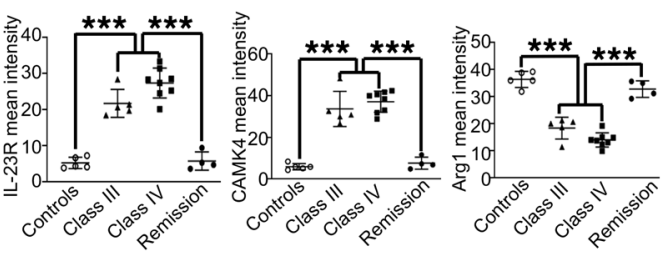


Figure 6. Upregulation of IL-23R and CaMK4 aligned with the reduction of ARG1 in renal biopsies from patients with glomerulonephritis. Human proximal TECs were cultured in vitro and stimulated with or without IL-23. (A) Left: Representative mean ECAR of TECs prestimulated with IL-23 for 6 hours (PBS as control, blue) with (green) or without (red) addition of EGTA. Dot plots represent cumulative data of calculated glycolysis (middle) and glycolytic capacity (right). (B) Heatmap of customized PCR array showing differential expression of 22 genes in TECs stimulated with IL-23 for the indicated hours. (C) Western blots (left) and quantification (right) of the expression of the indicated proteins in TECs stimulated with PBS or IL-23 for 48 hours. (E) Flow cytometric analysis of in vitro proliferative response (CFSE ${ }^{10}$ ) of purified $C D 4^{+} T$ cells from healthy PBMCs cocultured with TECs or IL-23-prestimulated TECs in arginine-deficient media with or without addition of arginine or citrulline for 72 hours $(n=4)$. T cells were stimulated with anti-CD3 and anti-CD28. (F-H) Cryosection of human kidney biopsies from the indicated group (control: healthy donor, $n=5$; AR: allograft rejection, $n=7$; ANCA: anti-neutrophil cytoplasmic autoantibody-associated glomerulonephritis, $n=4$; LN: active lupus nephritis, $n=13$ ). (I-K) Cryosection of human kidney biopsies from patients with indicted types of lupus nephritis (healthy donor, $n=5$; class III lupus nephritis, $n=5$; class IV lupus nephritis, $n=8$; lupus nephritis with remission status, $n=4)$. ( $F$ and $I$ ) Renal biopsies stained for synaptopodin (blue), IL-23R (green), and CaMK4 (red). Magnification, $\times 4$; scale bar: $180 \mu \mathrm{m}$. Top: Synaptopodin and IL-23R. Bottom: Synaptopodin and CaMK4. (G and J). Renal interstitium stained for CD26 (blue), ARG1 (red), and IL-23R (upper, green) or CaMK4 (bottom, green). (H) ImageJ intensity quantification plots. Magnification, $\times 20$; scale bar: $50 \mu \mathrm{m}$. Data represent the mean $\pm \mathrm{SEM} .{ }^{*} P<0.05,{ }^{* *} P<0.005$ versus control by Student's $t$ test (A and $\mathbf{D}$ ) or 2-way ANOVA (H and $\mathbf{K}$ ).

CaMK2 antibody (clone 45, BD Biosciences), separately diluted in PBS. HRP-conjugated goat anti-mouse secondary antibody was applied for 20 minutes. DAB substrate reagent (catalog 34001, Life Technologies) was prepared immediately before use and added for 7 minutes. The stained sections were lightly counterstained with hematoxylin before mounting. In all cases, negative controls, consisting of incubations without the primary antibody, were included in the analyses.

Histopathological analysis. Kidneys were cut in half longitudinally, embedded in paraffin, sectioned onto slides, stained with H\&E, examined microscopically, and scored as follows: (a) presence of crescents or fibrosis in glomeruli (1\%-10\%, grade $1 ; 10 \%-25 \%$, grade $2 ; 25 \%-50 \%$, grade $3 ;>50 \%$, grade 4$)$; (b) tubular cell changes $(1 \%-10 \%$, grade 1 ; $10 \%-25 \%$, grade $2 ; 25 \%-50 \%$, grade $3 ;>50 \%$, grade 4 ); (c) lymphocyte infiltration (1\%-10\% of renal parenchyma, grade $1 ; 10 \%-25 \%$, grade 2 ; $25 \%-50 \%$, grade $3 ;>50 \%$, grade 4 ); and (d) vasculitis (grade 1 , small but definite perivascular infiltrates; grade 2, 1-3 foci of perivascular infiltrates without necrosis; grade $3,3-5$ foci of perivascular infiltrate, more extensive; grade 4 , more than 5 foci of perivascular infiltrates). The sum of the 4 histopathology scores was calculated (68).

Western blotting. TECs were lysed in RIPA buffer at $4^{\circ} \mathrm{C}$ for 30 minutes. After centrifugation $\left(16,400 \mathrm{~g}, 30\right.$ minutes, $\left.4^{\circ} \mathrm{C}\right)$, supernatants were collected and an identical amount of protein from each lysate was resolved in NuPAGE 4\%-12\% Bis-Tris gels (Thermo Fisher Scientific). Proteins were transferred to a nitrocellulose membrane, which was subsequently blocked for 1 hour using $5 \%$ nonfat dry milk in TBS-T and incubated at $4^{\circ} \mathrm{C}$ overnight with mouse anti-human/mouse CaMK4 (clone 26, BD Biosciences), mouse anti-human/mouse ARG1 (clone O94E5, BD BioLegend), goat anti-human/mouse IL-23R (catalog number ab53656, Abcam), or mouse anti-mouse/human $\beta$-actin (catalog C4, Santa Cruz Biotechnology). The membrane was washed with TBS-T and incubated with anti-rabbit, anti-goat, anti-mouse, or anti-sheep IgG antibody coupled with HRP (Santa Cruz Biotechnology) or mouse anti-rabbit IgG (conformation specific) with HRP (Cell Signaling Technology). The ECL system (Amersham) was used for detection. Bands on blots corresponding to proteins of interest were analyzed by ImageJ software (NIH).

Flow cytometric analysis and sorting. Excised spleens or kidneys were minced and digested for 30 minutes in RPMI (Gibco) containing $20 \%$ heat-inactivated FBS, $100 \mathrm{U} / \mathrm{mL}$ penicillin, $50 \mu \mathrm{g} / \mathrm{mL}$ streptomycin, $2 \mathrm{mM}$ glutamine, $50 \mu \mathrm{g} / \mathrm{mL}$ DNase I, and $0.5 \mathrm{mg} / \mathrm{mL}$ collagenase D. The tissue suspensions were centrifuged and RBCs in the cell pellets were lysed with ACK lysis buffer. The dissociated cell suspensions were strained through a $100-\mu \mathrm{m}$ sieve and the total cell numbers were counted. Cells were first stained at $4^{\circ} \mathrm{C}$ in PBS containing 2\% FCS after Fc $\gamma$ $\mathrm{RII} / \mathrm{III}$ blocking and then surface staining was performed with the following antibodies: anti-Thy1.2 (clone 53-2.1), anti-CD4 (clone GK1.5), anti-Gr-1 (clone RB6-8C5, BioLegend), and anti-Thy1.1 (clone OX-7). For intracellular analysis of cytokines, cells were stimulated for 5 hours with phorbol myristate acetate $(50 \mathrm{ng} / \mathrm{mL}$; Sigma-Aldrich) and ionomycin (750 ng/mL; Sigma-Aldrich) in the presence of GolgiPlug (BD Pharmingen) at the recommended concentrations. Cells were stained extracellularly first and then fixed and permeabilized with Cytofix/ Cytoperm solution (BD Pharmingen) before intracellular staining with anti-IFN- $\gamma$ (clone XMG1.2) and anti-IL-17 (clone TC11-18H10.1). The absolute cell numbers of populations of interest were calculated by multiplying the total cell number with related percentages achieved by flow cytometry. All antibodies were from BioLegend. Dead cells were excluded using Fixable Viability Dye staining (Life Technologies).

TECs and cell culture. Human primary renal proximal TECs were purchased from ATCC (PCS-400-010) and cells were grown in renal epithelial cell basal medium (PCS-400-030) supplemented with a renal epithelial cell growth kit (PCS-400-040). Mouse primary renal proximal TECs were isolated and enriched as described previously (39). Renal cortices were dissected and digested with collagenase at $37^{\circ} \mathrm{C}$ for 30 minutes. After digestion, the supernatant was passed through nylon sieves with different sizes (range from 50 to $110 \mu \mathrm{m}$ ) sequentially and TECs were resuspended and enriched in the culture medium: DMEM supplemented with epidermal growth factor (EGF) (10 ng/mL) and insulin (5 mg/mL).

Cell coculture assay. In vitro-cultured murine or human primary proximal TECs were further cocultured with purified and CFSE-labeled ( $1 \mathrm{mM})$ naive CD4 ${ }^{+} \mathrm{T}$ cells (50:1 ratio) in L-arginine-free RPMI 1640 medium (89984, Thermo Fisher Scientific). L-Arginine $(100 \mu \mathrm{M})$ alone was added or citrulline $(200 \mu \mathrm{M})$ was added with a low concentration of L-arginine $(25 \mu \mathrm{M})$. T cells were activated with plate-bound anti-CD3 $(5 \mu \mathrm{g} / \mathrm{mL}$, BioLegend) and anti-CD28 (1 $\mu \mathrm{g} / \mathrm{mL}$, BioLegend) for 72 hours and $\mathrm{T}$ cell proliferative responses were determined by the reduction in CFSE intensity by flow cytometry.

Metabolism assays. ECAR and OCR were assessed on Seahorse XF Analyzers using an Agilent Seahorse XF Glycolysis Stress Test kit (103020-100) and Agilent Seahorse XF Cell Mito Stress Test Kit (103015-100), respectively. All procedures were performed according to the manufacturer's instructions.

Calcium flux assay. Five million adherent TECs were detached and resuspended in RPMI 1640 supplemented with 1\% FBS. Cells were further loaded with indo-1 acetoxymethyl ester (Life Technologies) for 30 minutes at $37^{\circ} \mathrm{C}$ and washed 3 times with RPMI 1640 . Unstimulated samples were assessed first to achieve a baseline fluo- 
rescence ratio representing the concentration of intracellular calcium in the resting state and then cells were stimulated with $5 \mathrm{ng} / \mathrm{mL}$ IL-23 to record free intracytoplasmic calcium concentrations represented by the mean fluorescence ratio of violet to blue indo-1 fluorescence. Stimulation with ionomycin $(25 \mu \mathrm{g} / \mathrm{mL})$ was used as a positive control at the end of experiment. Data were acquired on a BD LSR II.

Autoantibody detection. Each ELISA well was coated with $5 \mathrm{mg}$ / $\mathrm{mL}$ of the test autoantigen (69). BiP was purchased from Assay Designs and all other protein autoantigens were purchased from Sigma-Aldrich. The assays were developed with an HRP-labeled isotype-specific goat anti-mouse antibody (Southern Biotechnology) and tetramethylbenzidine substrate (Sigma-Aldrich). The absorbance at $450 \mathrm{~nm}$ (A450) was measured with an EMax Microplate Reader (Molecular Devices).

Renal functional analysis. Spot urine samples were collected. Urinary albumin and creatinine were quantified using ELISA kits (Bethyl Laboratories and R\&D Systems, respectively). $\beta$-2-Microglobulin was captured by rat anti-mouse $\beta$-2-microglobulin antibody (clone A16041A, BioLegend) and detected by HRP-conjugated mouse antimouse/human $\beta$-2-microglobulin antibody (clone BBM.1, Santa Cruz Biotechnology). The recombinant mouse $\beta$-2-microglobulin standard was purchased from Sino Biological.

Isolation of TECs by FACS. Preparation of renal single-cell suspensions was performed as described previously (38). Single-cell suspensions were stained for kidney-resident cells using anti-mouse CD26 (BioLegend), nephrin (Bioss), CD31 (BioLegend), or CD45 (eBioscience). All FACS analyses were done on a BD FACSAria.

Nanolipogel preparations and characterization. KN93-loaded nanolipogels were made as described previously $(55,68)$. To achieve targeting for podocytes, nanolipogels were incubated with biotin-conjugated anti-mouse cadherin-16 antibody (CDH16/1071, Novus Biologicals) for 20 minutes at room temperature before injection. For analyzing targeting specificity of nanolipogels in vivo, anti-mouse cadherin-16coated rhodamine-labeled nanolipogels were i.v. administered.
Statistics. All results are shown as the mean \pm SEM. Statistical significance was determined by $t$ test (2-tailed) for 2 groups, or 2-way ANOVA with Bonferroni's multiple-comparison test for 3 or more groups. $P$ values of less than 0.05 were considered significant.

Study approval. All animal procedures were approved by the Institutional Animal Care and Use Committee of Beth Israel Deaconess Medical Center, Harvard Medical School. The kidney biopsy samples used in this study were obtained from Department of Pathology, Beth Israel Deaconess Medical Center, Harvard Medical School (IRB number 2006P000298).

\section{Author contributions}

HL and GCT designed the experiments, analyzed the data, and wrote the manuscript. HL performed the experiments. GCT secured resources. MGT and RB reviewed all kidney section slides and provided the histopathology scores. IEA provided the minicircles and PR provided the $I l 23 \mathrm{r}^{f / f l}$ mice. HMJ and IES provided the human biopsies along with clinical information. JJ provided expert guidance. All authors reviewed the manuscript.

\section{Acknowledgments}

This worked was supported by grants from the NIH (R01 AI085567 to GCT and T32 DK007199 to HL) and a grant from Janssen. PR is supported by the DFG EXC "Precision Medicine in Chronic Inflammation," the German Federal Ministry of Education and Research (BMBF) grant iTREAT (SP5), and the EU H2O2O grant SYSCID (contract no. 733100). We thank Tarek Fahmy for preparing KN93-loaded nanolipogels.

Address correspondence to: Hao Li, CLS-928, 330 Blackfan Circle, Boston, Massachusetts 02115, USA. Phone: 617.735.2578; Email: hli13@bidmc.harvard.edu. Or to: George C. Tsokos, CLS 37, 330 Blackfan Circle, Boston, Massachusetts 02115, USA. Phone: 617.735.4161; Email: gtsokos@bidmc.harvard.edu.
1. GBD Chronic Kidney Disease Collaboration. Global, regional, and national burden of chronic kidney disease, 1990-2017: a systematic analysis for the Global Burden of Disease Study 2017. Lancet. 2020;395(10225):709-733.

2. Schiffer $\mathrm{L}$, et al. Activated renal macrophages are markers of disease onset and disease remission in lupus nephritis. JImmunol. 2008;180(3):1938-1947.

3. Bethunaickan R, et al. A unique hybrid renal mononuclear phagocyte activation phenotype in murine systemic lupus erythematosus nephritis. JImmunol. 2011;186(8):4994-5003.

4. Davidson A. What is damaging the kidney in lupus nephritis? Nat Rev Rheumatol. 2016;12(3):143-153.

5. Chang A, et al. In situ B cell-mediated immune responses and tubulointerstitial inflammation in human lupus nephritis. J Immunol. 2011;186(3):1849-1860.

6. Liarski VM, et al. Cell distance mapping identifies functional $\mathrm{T}$ follicular helper cells in inflamed human renal tissue. Sci Transl Med. 2014;6(230):230ra46.

7. Suarez-Fueyo A, et al. T cells and autoimmune kidney disease. Nat Rev Nephrol. 2017;13(6):329-343.
8. Hsieh C, et al. Predicting outcomes of lupus nephritis with tubulointerstitial inflammation and scarring. Arthritis Care Res (Hoboken). 2011;63(6):865-874.

9. Stenvinkel P, et al. Strong association between malnutrition, inflammation, and atherosclerosis in chronic renal failure. Kidney Int. 1999;55(5):1899-1911.

10. Winchester R, et al. Immunologic characteristics of intrarenal T cells: trafficking of expanded $\mathrm{CD}^{+} \mathrm{T}$ cell $\beta$-chain clonotypes in progressive lupus nephritis. Arthritis Rheum. 2012;64(5):1589-1600.

11. Chen A, et al. Bowman's capsule provides a protective niche for podocytes from cytotoxic $\mathrm{CD}^{+}$ T cells. J Clin Invest. 2018;128(8):3413-3424.

12. Li H, et al. Systemic lupus erythematosus favors the generation of IL-17 producing double negative T cells. Nat Commun. 2020;11(1):2859.

13. Chen PM, et al. Kidney tissue hypoxia dictates $\mathrm{T}$ cell-mediated injury in murine lupus nephritis. Sci Transl Med. 2020;12(538): eaay1620.

14. Weyand CM, Goronzy JJ. Immunometabolism in early and late stages of rheumatoid arthritis. Nat Rev Rheumatol. 2017;13(5):291-301.
15. Weyand CM, et al. Metabolic signatures of T-cells and macrophages in rheumatoid arthritis. Curr Opin Immunol. 2017;46:112-120.

16. Yin $\mathrm{Y}$, et al. Normalization of $\mathrm{CD}^{+} \mathrm{T}$ cell metabolism reverses lupus. Sci Transl Med. 2015;7(274):274ra18.

17. Boyman O, et al. Homeostatic maintenance of T cells and natural killer cells. Cell Mol Life Sci. 2012;69(10):1597-1608.

18. Sharabi A, Tsokos GC. T cell metabolism: new insights in systemic lupus erythematosus pathogenesis and therapy. Nat Rev Rheumatol. 2020;16(2):100-112.

19. Buck MD, et al. T cell metabolism drives immunity. JExp Med. 2015;212(9):1345-1360.

20. Kedia-Mehta N, Finlay DK. Competition for nutrients and its role in controlling immune responses. Nat Commun. 2019;10(1):2123.

21. de Haij S, et al. Renal tubular epithelial cells modulate T-cell responses via ICOS-L and B7-H1. Kidney Int. 2005;68(5):2091-2102.

22. Teng MW, et al. IL-12 and IL-23 cytokines: from discovery to targeted therapies for immunemediated inflammatory diseases. Nat Med. 2015;21(7):719-729. 
23. Gaffen SL, et al. The IL-23-IL-17 immune axis: from mechanisms to therapeutic testing. Nat Rev Immunol. 2014;14(9):585-600.

24. Crispin JC, et al. Expanded double negative T cells in patients with systemic lupus erythematosus produce IL-17 and infiltrate the kidneys. J Immunol. 2008;181(12):8761-8766

25. MacDonald KP, et al. Cytokine mediators of chronic graft-versus-host disease. JClin Invest. 2017;127(7):2452-2463.

26. Paust HJ, et al. The IL-23/Th17 axis contributes to renal injury in experimental glomerulonephritis. JAm Soc Nephrol. 2009;20(5):969-979.

27. Zhang Z, et al. The role of IL-23/IL-17 axis in lupus nephritis. JImmunol. 2009;183(5):3160-3169.

28. Racioppi L, Means AR. Calcium/calmodulindependent kinase IV in immune and inflammatory responses: novel routes for an ancient traveller. Trends Immunol. 2008;29(12):600-607.

29. Bronte V, Murray PJ. Understanding local macrophage phenotypes in disease: modulating macrophage function to treat cancer. Nat Med. 2015;21(2):117-119.

30. Bronte $\mathrm{V}$, et al. L-arginine metabolism in myeloid cells controls T-lymphocyte functions. Trends Immunol. 2003;24(6):302-306.

31. Geiger R, et al. L-arginine modulates T cell metabolism and enhances survival and antitumor activity. Cell. 2016;167(3):829-842.

32. Biswas PS. IL-17 in renal immunity and autoimmunity. J Immunol. 2018;201(11):3153-3159.

33. Adamopoulos IE, et al. IL-23 is critical for induction of arthritis, osteoclast formation, and maintenance of bone mass. Jimmunol. 2011;187(2):951-959.

34. Sherlock JP, et al. IL-23 induces spondyloarthropathy by acting on ROR- $\gamma \mathrm{t}^{+} \mathrm{CD} 3^{+} \mathrm{CD} 4{ }^{-\mathrm{CD}} 8$ - entheseal resident T cells. Nat Med. 2012;18(7):1069-1076.

35. Leys L, et al. Characterization of psoriasiform dermatitis induced by systemic injection of interleukin-23 minicircles in mice. J Dermatol. 2019;46(6):482-497.

36. Peterson PA, et al. Differentiation of glomerular, tubular, and normal proteinuria: determinations of urinary excretion of beta-2-macroglobulin, albumin, and total protein. JClin Invest. 1969;48(7):1189-1198.

37. Ebert T, et al. Establishment and characterization of human renal cancer and normal kidney cell lines. Cancer Res. 1990;50(17):5531-5336.

38. Fu R, et al. Podocyte activation of NLRP3 inflammasomes contributes to the development of proteinuria in lupus nephritis. Arthritis Rheumatol. 2017;69(8):1636-1646.

39 . Terryn S, et al. A primary culture of mouse proximal tubular cells, established on collagen-coat- ed membranes. Am J Physiol Renal Physiol. 2007;293(2):F476-F485.

40. Spiegel DM, Brady K. Calcium balance in normal individuals and in patients with chronic kidney disease on low- and high-calcium diets. Kidney Int. 2012;81(11):1116-1122.

41. Yamaguchi T, et al. Calcium restores a normal proliferation phenotype in human polycystic kidney disease epithelial cells. J Am Soc Nephrol. 2006;17(1):178-187.

42. Weichhart T, et al. Regulation of innate immune cell function by mTOR. Nat Rev Immunol. 2015;15(10):599-614.

43. O'Neill LA, et al. A guide to immunometabolism for immunologists. Nat Rev Immunol. 2016;16(9):553-565.

44. Tokumitsu H, et al. Calcium/calmodulin-dependent protein kinase kinase: identification of regulatory domains. Biochemistry. 1997;36(42):12823-12827.

45. McGeachy MJ, et al. The interleukin 23 receptor is essential for the terminal differentiation of interleukin 17-producing effector T helper cells in vivo. Nat Immunol. 2009;10(3):314-324.

46. Mondanelli G, et al. Amino acid metabolism as drug target in autoimmune diseases. Autoimmun Rev. 2019;18(4):334-348.

47. Munder M, et al. Suppression of T-cell functions by human granulocyte arginase. Blood. 2006;108(5):1627-1634.

48. Werner A, et al. Reconstitution of T cell proliferation under arginine limitation: activated human T cells take up citrulline via L-type amino acid transporter 1 and use it to regenerate arginine after induction of argininosuccinate synthase expression. Front Immunol. 2017;8:864.

49. Koga T, et al. KN-93, an inhibitor of calcium/ calmodulin-dependent protein kinase IV, promotes generation and function of Foxp $3^{+}$regulatory T cells in MRL/lpr mice. Autoimmunity. 2014;47(7):445-450.

50. Shao X, et al. Epithelial-specific Cre/lox recombination in the developing kidney and genitourinary tract. J Am Soc Nephrol. 2002;13(7):1837-1846.

51. Salant DJ, Cybulsky AV. Experimental glomerulonephritis. Methods Enzymol. 1988;162:421-461.

52. Tsokos GC. Systemic lupus erythematosus. $N$ Engl J Med. 2011;365(22):2110-2121.

53. Andrews BS, et al. Spontaneous murine lupus-like syndromes. Clinical and immunopathological manifestations in several strains. JExp Med. 1978;148(5):1198-1215.

54. Kyttaris VC, et al. Cutting edge: IL-23 receptor deficiency prevents the development of lupus nephritis in C57BL/6-lpr/lpr mice. J Immunol. 2010;184(9):4605-4609.

55. Otomo K, et al. Cutting edge: nanogel-based delivery of an inhibitor of CaMK 4 to $\mathrm{CD} 4^{+} \mathrm{T}$ cells suppresses experimental autoimmune encephalomyelitis and lupus-like disease in mice. JImmunol. 2015;195(12):5533-5537.

56. Maeda K, et al. CaMK4 compromises podocyte function in autoimmune and nonautoimmune kidney disease. JClin Invest. 2018;128(8):3445-3459.

57. Thomson RB, Aronson PS. Immunolocalization of Ksp-cadherin in the adult and developing rabbit kidney. Am J Physiol.1999;277(1):F146-F156.

58. Li H, et al. IL-23 promotes TCR-mediated negative selection of thymocytes through the upregulation of IL-23 receptor and ROR $\gamma \mathrm{t}$. Nat Commun. 2014;5:4259.

59. Rosenblum MD, et al. Treating human autoimmunity: current practice and future prospects. Sci Transl Med. 2012;4(125):125sr1.

60. Yu F, et al. Tubulointerstitial lesions of patients with lupus nephritis classified by the 2003 International Society of Nephrology and Renal Pathology Society system. Kidney Int. 2010;77(9):820-829.

61. van Vollenhoven RF, et al. Efficacy and safety of ustekinumab, an IL-12 and IL-23 inhibitor, in patients with active systemic lupus erythematosus: results of a multicentre, double-blind, phase 2, randomised, controlled study. Lancet. 2018;392(10155):1330-1339.

62. Ma C, et al. Investigational drugs in phase I and phase II clinical trials targeting interleukin 23 (IL23) for the treatment of Crohn's disease. Expert Opin Investig Drugs. 2018;27(8):649-660.

63. Amin M, et al. Review of phase III trial data on IL-23 inhibitors tildrakizumab and guselkumab for psoriasis. J Eur Acad Dermatol Venereol. 2017;31(10):1627-1632.

64. Awasthi A, et al. Cutting edge: IL-23 receptor gfp reporter mice reveal distinct populations of IL-17-producing cells. J Immunol. 2009;182(10):5904-5908.

65. Aden K, et al. Epithelial IL-23R signaling licenses protective IL-22 responses in intestinal inflammation. Cell Rep. 2016;16(8):2208-2218.

66. Adamopoulos IE, et al. IL-17A gene transfer induces bone loss and epidermal hyperplasia associated with psoriatic arthritis. Ann Rheum Dis. 2015;74(6):1284-1292.

67. Ge Y, et al. Cgnz1 allele confers kidney resistance to damage preventing progression of immune complex-mediated acute lupus glomerulonephritis. J Exp Med. 2013;210(11):2387-2401.

68. Li H, et al. Precision DNA demethylation ameliorates disease in lupus-prone mice. JCI Insight. 2018;3(16):e120880.

69. $\mathrm{Li} \mathrm{H}$, et al. Interferon-induced mechanosensing defects impede apoptotic cell clearance in lupus. J Clin Invest. 2015;125(7):2877-2890. 